\title{
Correlation and Capacity Calculations with Reference Antennas in an Isotropic Environment
}

\author{
Thorkild B. Hansen \\ Consultant, Azimuth Systems, Inc., 35 Nagog Park, Acton, MA 01720, USA \\ Correspondence should be addressed to Thorkild B. Hansen, thorkild.hansen@att.net \\ Received 5 January 2012; Revised 10 March 2012; Accepted 21 March 2012 \\ Academic Editor: Moray Rumney
}

Copyright () 2012 Thorkild B. Hansen. This is an open access article distributed under the Creative Commons Attribution License, which permits unrestricted use, distribution, and reproduction in any medium, provided the original work is properly cited.

\begin{abstract}
A reverberation chamber is a convenient tool for over-the-air testing of MIMO devices in isotropic environments. Isotropy is typically achieved in the chamber through the use of a mode stirrer and a turntable on which the device under test (DUT) rides. The quality of the isotropic environment depends on the number of plane waves produced by the chamber and on their spatial distribution. This paper investigates how the required sampling rate for the DUT pattern is related to the plane-wave density threshold in the isotropic environment required to accurately compute antenna correlations. Once the plane-wave density is above the threshold, the antenna correlation obtained through isotropic experiments agrees with the antenna correlation obtained from the classical definition, as has been proven theoretically. This fact is verified for the good, nominal, and bad reference antennas produced by CTIA. MIMO channel capacity simulations are performed with a standard base station model and the DUT placed in a single-tap plane-wave reverberation chamber model. The capacity curves obtained with the good, nominal, and bad reference antennas are clearly distinguishable.
\end{abstract}

\section{Introduction}

The reverberation chamber [1] is a cost-efficient and convenient tool for creating an isotropic environment in which wireless devices can be tested. The field in a rectangular reverberation chamber can be described in terms of chamber modes that each can be expressed as a sum of eight plane waves $[2,3]$. The chamber typically contains a mode stirrer and a turn table on which the device under test (DUT) rides. At any fixed turntable and stirrer position, the DUT sees a certain collection of plane waves. By rotating the stirrer and turntable, a new collection of plane waves illuminates the DUT (the turntable causes the DUT to see each plane wave from different angles). The isotropic test environment is thus achieved by rotating both the stirrer and turntable to illuminate the DUT with many different collections of plane waves. One can determine if a given reverberation chamber at a given frequency is isotropic by evaluating anisotropy coefficients obtained from three-axis dipole experiments [23, Annex J] and [4].

In the present paper we investigate the use of reverberation chambers for over-the-air testing of MIMO devices.
Both antenna correlation (a quantity that is critically important for MIMO system performance) and MIMO capacity will be simulated in a plane-wave reverberation chamber model [5-9]. The simulations will be performed with a pair of Hertzian dipoles and with good, nominal, and bad reference antennas [10]. The classical antenna correlation is compared to the isotropic antenna correlation, and it is verified numerically that the two are equivalent, as proven by De Doncker and Meys [11].

To ensure accurate and reliable over-the-air test results, the test system must produce an accurate test environment in the entire physical region that contains the DUT. For example, in conventional 2D anechoic tests, the number of antennas in the ring surrounding the DUT must be large enough to accurately reproduce the desired channel conditions in the region occupied by the DUT. Similarly, the reverberation chamber must be large enough to supply enough plane waves to achieve an isotropic environment in the region occupied by the DUT.

By expressing the DUT pattern in terms of a spherical expansion (with a recently derived formula for truncation limit), we determine how closely the DUT pattern must 
be sampled to properly capture its variation. Using this sampling rate, we obtain rules that determine both the required number of antennas in the anechoic test and the required plane-wave density in the reverberation chamber test. As a byproduct of this investigation, we present accurate Fourier expansions of the DUT pattern that have been used in spherical near-field scanning for many years but appear to be relatively unknown in wireless communications.

The paper is organized as follows. Section 2 introduces the truncated spherical-wave expansion and derives the sampling theorems and Fourier expansions for the pattern of an arbitrary DUT. Section 3 deals with antenna correlations in both the reverberation chamber test and in a 2D anechoic chamber test. This section also relates the accuracy of simulated correlations to the sampling rate required for the pattern. In Section 4 we investigate the plane-wave distribution for realistic reverberation chambers by using the dyadic Green's function for the rectangular box. We further simulate antenna correlations and compute anisotropy coefficients. Section 5 presents MIMO channel capacity simulations using a standard base station model and the plane-wave reverberation chamber model. Section 6 presents conclusions. Throughout, we assume time-harmonic fields that have $e^{-i \omega t}$ time dependence with $\omega>0$.

\section{Plane-Wave Receiving Characteristic and Far-Field Pattern}

In this section we introduce the plane-wave receiving characteristic and far-field pattern of an arbitrary DUTmounted antenna. (The term "pattern" will be used to refer to both the plane-wave receiving characteristic and to the farfield antenna pattern). A spherical expansion determines the spatial sampling rate required to "capture" the pattern of the antenna and provides a Fourier series expansion useful for computing any quantity involving the pattern. The standard spherical coordinates $(r, \theta, \phi)$ with unit vectors given by

$$
\begin{gathered}
\hat{\mathbf{r}}(\theta, \phi)=\hat{\mathbf{x}} \cos \phi \sin \theta+\hat{\mathbf{y}} \sin \phi \sin \theta+\hat{\mathbf{z}} \cos \theta, \\
\hat{\boldsymbol{\theta}}(\theta, \phi)=\hat{\mathbf{x}} \cos \theta \cos \phi+\hat{\mathbf{y}} \cos \theta \sin \phi-\hat{\mathbf{z}} \sin \theta, \\
\\
\hat{\boldsymbol{\phi}}(\theta, \phi)=-\hat{\mathbf{x}} \sin \phi+\hat{\mathbf{y}} \cos \phi
\end{gathered}
$$

will be used throughout. Here, the unit vectors for the rectangular coordinates $(x, y, z)$ are $\hat{\mathbf{x}}, \hat{\mathbf{y}}$, and $\hat{\mathbf{z}}$. Note that $\widehat{\mathbf{r}}(\theta, \phi)$ with $0 \leq \theta \leq \pi$ and $0 \leq \phi<2 \pi$ covers the unit sphere once. Figure 1 shows the spherical coordinates.

The DUT with a mounted antenna is shown in Figure 2 inside the minimum sphere with radius $R_{\min }$, defined such that the maximum value of the coordinate $r$ for all points on the DUT equals $R_{\min }$. Note that $R_{\min }$ depends not only on the size of the DUT but also on its location with respect to the coordinate system. For example, a Hertzian dipole located at $\mathbf{r}_{0}$ has $R_{\min }=\left|\mathbf{r}_{0}\right|$ despite the fact that its physical extent is vanishing. Also, even if the physical dimension of a DUTmounted antenna is much smaller than the dimensions of the DUT (as in Figure 2), the antenna interacts with the DUT and therefore it is the entire DUT size that must be used when computing $R_{\min }$.

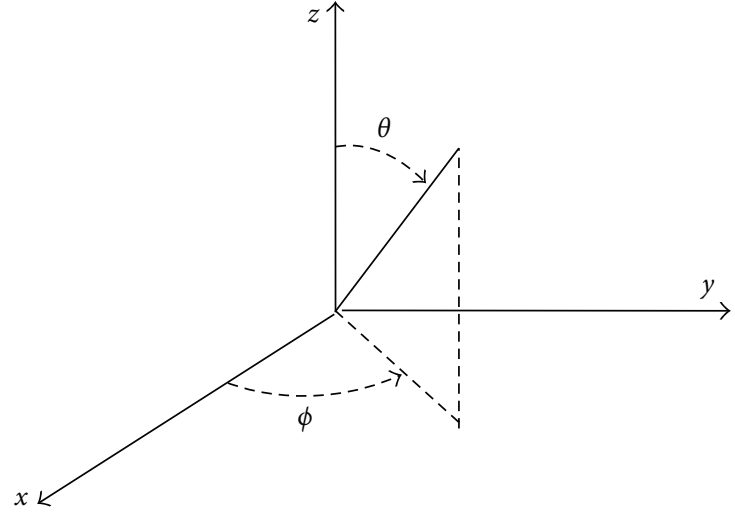

FIGURE 1: Spherical coordinates.

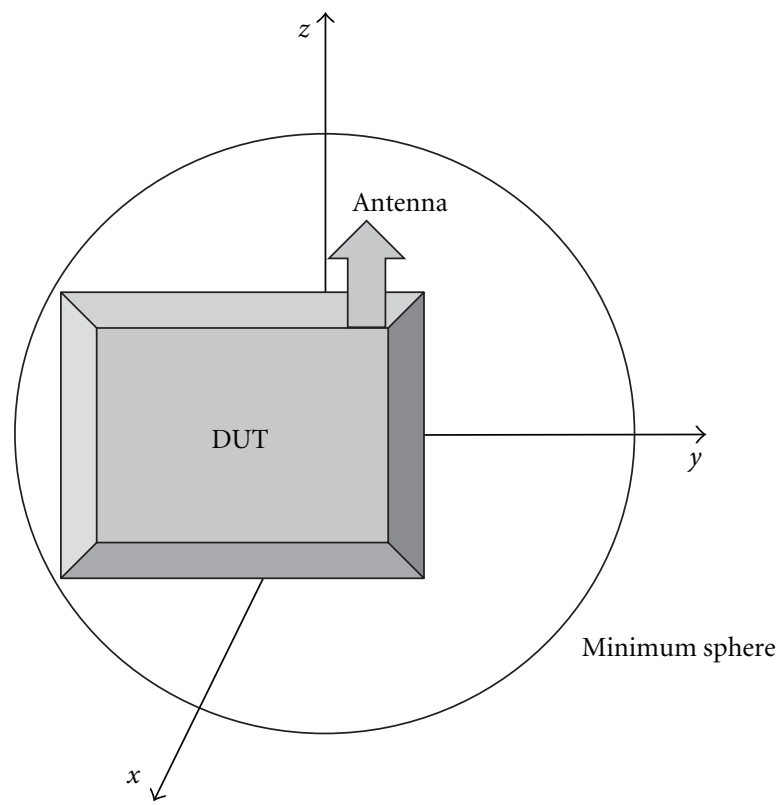

FIGURE 2: The DUT with mounted antenna contained in the minimum sphere of radius $R_{\min }$.

The plane-wave receiving characteristic is defined as follows. Assume that the incident plane wave $\mathbf{E}(\mathbf{r})=\mathbf{E}_{0} e^{i k \hat{\mathbf{k}}_{0} \cdot \mathbf{r}}$ with propagation direction $\hat{\mathbf{k}}_{0}$ illuminates the DUT. The direction of propagation is $\hat{\mathbf{k}}_{0}=-\widehat{\mathbf{r}}\left(\theta_{0}, \phi_{0}\right)$, and the constant vector $\mathbf{E}_{0}$ satisfies $\mathbf{E}_{0} \cdot \hat{\mathbf{k}}_{0}=0$. With this notation, the incident plane wave "comes" from the direction $\left(\theta_{0}, \phi_{0}\right)$. For example, if $\theta_{0}=0$, the plane wave is $\mathbf{E}(\mathbf{r})=\mathbf{E}_{0} e^{-i k z}$ and propagates in the direction of the negative $z$-axis. When the DUT is illuminated by this plane-wave field, its output is by definition $V=\mathscr{R}\left(\theta_{0}, \phi_{0}\right) \cdot \mathbf{E}_{0}$, where $\mathcal{R}\left(\theta_{0}, \phi_{0}\right)$ is the planewave receiving characteristic satisfying $\hat{\mathbf{k}}_{0} \cdot \mathcal{R}\left(\theta_{0}, \phi_{0}\right)=0$.

If the DUT-mounted antenna satisfies reciprocity, its plane-wave receiving characteristic can be expressed in terms of its normalized far-field pattern $\mathscr{F}_{0}(\theta, \phi)$ as $[12$, equation (6.60)] (The spherical angles determining the plane-wave 
directions of propagation in [12] are different from those used in the present paper):

$$
\mathcal{R}\left(\theta_{0}, \phi_{0}\right)=\sqrt{\frac{\epsilon}{\mu}} \frac{i}{k Y_{p}} \mathscr{F}_{0}\left(\theta_{0}, \phi_{0}\right),
$$

where $\mu$ and $\epsilon$ are the free-space permeability and permittivity, respectively. Moreover, $k=\omega \sqrt{\mu \epsilon}$ is the wavenumber and $Y_{p}$ is the characteristic admittance of the propagating mode of a wave-guide feed assumed attached to the DUT antenna; see [12, Chapter 6]. In general, if the antenna is not reciprocal, the receiving characteristic can be related to the pattern of an adjoint antenna [13].

The electric far field of the DUT, when it is fed by an input voltage-amplitude $V_{0}$, is [12, equation (6.35)] (The nonnormalized far-field pattern $\mathscr{F}(\theta, \phi)$ is defined in [12, equation (3.31)] in terms of the electric field through the limit $\mathscr{F}(\theta, \phi)=\lim _{r \rightarrow \infty} r e^{-i k r} \mathbf{E}(r, \theta, \phi)$. The normalized farfield pattern $\mathscr{F}_{0}(\theta, \phi)$ is in turn defined by $V_{0} \mathscr{F}_{0}(\theta, \phi)=$ $\mathscr{F}(\theta, \phi)$, where $V_{0}$ is the input voltage amplitude of the signal that feeds the antenna. Note that the symbol " $\sim$ " in (3) means "asymptotically equal to" in the limit $r \rightarrow \infty$.)

$$
\mathbf{E}(r, \theta, \phi) \sim V_{0} \widetilde{F}_{0}(\theta, \phi) \frac{e^{i k r}}{r} .
$$

The far-field pattern $\mathscr{\mathcal { F }}\left(\theta_{0}, \phi_{0}\right)$ determines the far field in the direction $\left(\theta_{0}, \phi_{0}\right)$ whereas $\mathcal{R}\left(\theta_{0}, \phi_{0}\right)$ determines the output due to a plane wave "coming in" from the direction $\left(\theta_{0}, \phi_{0}\right)$. Hence, this incident plane wave propagates in the direction $\left(\pi-\theta_{0}, \pi+\phi_{0}\right)$. Also, the normalized far-field pattern $\widetilde{F}_{0}(\theta, \phi)$ is dimensionless and the plane-wave receiving characteristic $\mathcal{R}(\theta, \phi)$ is a length.

These statements fully define the plane-wave receiving characteristic for any propagating plane wave that may illuminate the DUT. If the source of the incident field is close to the DUT, one must also specify the plane-wave receiving characteristic for evanescent plane waves [12, Chapters 3 and 6]. However, in this paper we consider only sources that are at least a few wavelengths away from the DUT so that evanescent waves are negligible.

Using (2) in conjunction with standard spherical-wave theory $[14,15]$ shows that the receiving characteristic can be expressed in terms of the transverse vector-wave functions $\mathbf{M}_{n m}$ and $\mathbf{N}_{n m}$ as

$$
\mathcal{R}(\theta, \phi)=\sum_{n=1}^{N} \sum_{m=-n}^{n}\left[A_{n m} \mathbf{M}_{n m}(\theta, \phi)+B_{n m} \mathbf{N}_{n m}(\theta, \phi)\right],
$$

where $A_{n m}$ and $B_{n m}$ are spherical expansion coefficients satisfying $A_{n m}=0$ and $B_{n m}=0$ when $|m|>n$. The truncation number $N$ is determined from the radius of the minimum sphere as

$$
N=\operatorname{int}\left(k R_{\min }+\gamma\left(k R_{\min }\right)^{1 / 3}\right)
$$

where the constant $\gamma$ determines the number of digits of accuracy achieved [16, Section 3.4.2] and "int" denotes the integral part. The formula (5) is especially useful for small sources where the second term is on the same order of magnitude as the first term. (In older literature the following truncation formula is often used with the second term left unspecified: " $N=\operatorname{int}\left(k R_{\min }+n_{1}\right)$ where $n_{1}$ is a small integer.")

The transverse vector-wave functions can be expressed in terms of the spherical harmonic $Y_{n m}(\theta, \phi)$ [15, page 99] as $[15$, pages $742-746]$

$$
\mathbf{M}_{n m}(\theta, \phi)=\hat{\boldsymbol{\theta}} \frac{i m Y_{n m}(\theta, \phi)}{\sqrt{n(n+1)} \sin \theta}-\hat{\boldsymbol{\phi}} \frac{(\partial / \partial \theta) Y_{n m}(\theta, \phi)}{\sqrt{n(n+1)}},
$$

and $\mathbf{N}_{n m}(\theta, \phi)=\hat{\mathbf{r}} \times \mathbf{M}_{n m}(\theta, \phi)$. The orthogonality relations [15] for the transverse vector-wave functions give the following well-known expressions for the spherical expansion coefficients $A_{n m}$ and $B_{n m}$ :

$$
\begin{aligned}
A_{n m} & =\int_{0}^{2 \pi} \int_{0}^{\pi} \mathcal{R}(\theta, \phi) \cdot \mathbf{M}_{n m}^{*}(\theta, \phi) \sin \theta d \theta d \phi, \\
B_{n m} & =\int_{0}^{2 \pi} \int_{0}^{\pi} \mathcal{R}(\theta, \phi) \cdot \mathbf{N}_{n m}^{*}(\theta, \phi) \sin \theta d \theta d \phi,
\end{aligned}
$$

where $*$ indicates complex conjugation. The formula (4) makes it possible to compute the plane-wave receiving characteristic in any direction from the spherical expansion coefficients $A_{n m}$ and $B_{n m}$. However, in this paper we shall use (4) to derive Fourier expansions and sampling theorems that are useful for computing quantities like correlations that involve the plane-wave receiving characteristic.

The expressions for the transverse vector-wave functions $\mathbf{M}_{n m}(\theta, \phi)$ and $\mathbf{N}_{n m}(\theta, \phi)$ show that the $\theta$ and $\phi$ components of $\mathcal{R}(\theta, \phi)=\mathcal{R}_{\theta}(\theta, \phi) \hat{\boldsymbol{\theta}}+\mathcal{R}_{\phi}(\theta, \phi) \hat{\boldsymbol{\phi}}$ in (4) have the Fourier series (The expressions [15, page 98] for the associated Legendre function show that both the derivative $(\partial / \partial \theta) Y_{n m}(\theta, \phi)$ and the fraction $m Y_{n m}(\theta, \phi) / \sin \theta$ can be expanded in terms of $e^{i m \phi} e^{i q \theta}$ with $q=-n, \ldots, n$.):

$$
\begin{aligned}
& \mathcal{R}_{\theta}(\theta, \phi)=\sum_{q=-N}^{N} \sum_{m=-N}^{N} \mathcal{D}_{q m}^{\theta} e^{i m \phi} e^{i q \theta}, \\
& \mathcal{R}_{\phi}(\theta, \phi)=\sum_{q=-N}^{N} \sum_{m=-N}^{N} \mathcal{D}_{q m}^{\phi} e^{i m \phi} e^{i q \theta},
\end{aligned}
$$

where $\mathscr{D}_{q m}^{\theta}$ and $\mathscr{D}_{q m}^{\phi}$ are Fourier coefficients. The Fourier expansions (8) define functions that are $2 \pi$-periodic in both $\theta$ and $\phi$. Hence, the Fourier coefficients cannot be determined from the sampling theorem for periodic spatially bandlimited functions when $\mathcal{R}(\theta, \phi)$ is known only over the standard sphere $0 \leq \theta \leq \pi, 0 \leq \phi \leq 2 \pi$. We shall overcome this problem by continuing $\mathcal{R}(\theta, \phi)$ to the interval $0 \leq \theta \leq 2 \pi$ (see $[17,18]$, and [19, pages 111-113, 140-144]).

Since $\widehat{\mathbf{r}}(\theta, \phi)=\widehat{\mathbf{r}}(2 \pi-\theta, \phi+\pi)$, the two points $(\theta, \phi)$ and $(2 \pi-\theta, \phi+\pi)$ correspond to the same point in space. Moreover, since the tangential spherical unit vectors satisfy $\hat{\boldsymbol{\theta}}(\theta, \phi)=-\hat{\boldsymbol{\theta}}(2 \pi-\theta, \phi+\pi)$ and $\hat{\boldsymbol{\phi}}(\theta, \phi)=-\hat{\boldsymbol{\phi}}(2 \pi-\theta, \phi+\pi)$, 
we can analytically continue $\mathcal{R}(\theta, \phi)$ into a $2 \pi$-periodic in both $\theta$ and $\phi$ by use of

$$
\begin{aligned}
& \mathcal{R}_{\theta}(\theta, \phi)=-\mathcal{R}_{\theta}(2 \pi-\theta, \phi+\pi), \\
& \mathcal{R}_{\phi}(\theta, \phi)=-\mathcal{R}_{\phi}(2 \pi-\theta, \phi+\pi) .
\end{aligned}
$$

One can show that the conditions (9) imply that the Fourier coefficients satisfy $\mathscr{D}_{q m}^{\theta}=(-1)^{m+1} \mathscr{D}_{-q, m}^{\theta}$ and $\mathscr{D}_{q m}^{\phi}=$ $(-1)^{m+1} \mathscr{D}_{-q, m}^{\phi}$.

Assume that $\mathcal{R}_{\theta}(\theta, \phi)$ and $\mathcal{R}_{\phi}(\theta, \phi)$ are known over the standard sphere $0 \leq \theta \leq \pi, 0 \leq \phi \leq 2 \pi$ at $\theta=(t-1) \Delta \theta$, $t=1, \ldots, N_{\theta}$ and $\phi=(p-1) \Delta \phi, p=1, \ldots, N_{\phi}$, where the sample rates are $\Delta \theta=\pi /\left(N_{\theta}-1\right)$ and $\Delta \phi=2 \pi / N_{\phi}$, with $N_{\theta} \geq$ $N+2$ and $N_{\phi} \geq 2 N+1$. Then the Fourier coefficients can be computed from the sampling theorem for periodic spatially bandlimited functions in conjunction with (9) as [19] and [20, Section IV]:

$$
\begin{aligned}
\mathscr{D}_{q m}^{\theta}= & \frac{1}{N_{\phi}\left(2 N_{\theta}-2\right)} \sum_{p=1}^{N_{\phi}} e^{-i m(p-1) \Delta \phi} \\
\times & {\left[\sum_{t=1}^{N_{\theta}} \mathcal{R}_{\theta}([t-1] \Delta \theta,[p-1] \Delta \phi) e^{-i q(t-1) \Delta \theta}\right.} \\
& \left.+(-1)^{m+1} \sum_{t=2}^{N_{\theta}-1} \mathcal{R}_{\theta}([t-1] \Delta \theta,[p-1] \Delta \phi) e^{i q(t-1) \Delta \theta}\right], \\
\mathcal{D}_{q m}^{\phi}= & \frac{1}{N_{\phi}\left(2 N_{\theta}-2\right)} \sum_{p=1}^{N_{\phi}} e^{-i m(p-1) \Delta \phi} \\
& \times\left[\sum_{t=1}^{N_{\theta}} \mathcal{R}_{\phi}([t-1] \Delta \theta,[p-1] \Delta \phi) e^{-i q(t-1) \Delta \theta}\right. \\
& \left.+(-1)^{m+1} \sum_{t=2}^{N_{\theta}-1} \mathcal{R}_{\phi}([t-1] \Delta \theta,[p-1] \Delta \phi) e^{i q(t-1) \Delta \theta}\right] .
\end{aligned}
$$

Of course, (10) hold only for functions that satisfy (9). We summarize the results (which also hold for the antenna pattern $\left.\widetilde{F}_{0}(\theta, \phi)\right)$ as follows.

(i) The plane-wave receiving characteristic $\mathcal{R}(\theta, \phi)$ should be sampled over the sphere $0 \leq \theta \leq \pi$ and $0 \leq \phi<2 \pi$ at a rate of at least $\Delta \theta=\Delta \phi=\pi /(N+$ 1 ), where $N$, given by (5), depends on frequency, physical DUT size, and DUT location.

(ii) The plane-wave receiving characteristic $\mathcal{R}(\theta, \phi)$ can be expressed in terms of the Fourier series (8) with Fourier coefficients computed through (10) from sampled values of $\mathcal{R}(\theta, \phi)$.

(iii) Integrals of the form

$$
I=\int_{0}^{2 \pi} \int_{0}^{\pi} \mathbf{U}(\theta, \phi) \cdot \mathcal{R}(\theta, \phi) \sin \theta d \theta d \phi,
$$

where $\mathbf{U}(\theta, \phi)$ is a known function, occur in many places. For example, the expressions (7) for the spherical expansion coefficients have this form. Such integrals can be computed accurately by inserting the Fourier expansions (8) for $\mathcal{R}(\theta, \phi)$. One can often compute the contribution from each Fourier term explicitly. Alternatively, by use of the Fourier series one can resample $\mathcal{R}(\theta, \phi)$ to a finer grid and then compute $I$ through numerical integration.

(iv) In contrast, brute-force approximations of the form (with the original sampling rate retained)

$$
\begin{aligned}
I \simeq \sum_{t=1}^{N_{\theta}} \sum_{p=1}^{N_{\phi}} \mathbf{U}([t-1] \Delta \theta,[p-1] \Delta \phi) \\
\quad \times \mathscr{R}([t-1] \Delta \theta,[p-1] \Delta \phi) \\
\quad \times \sin ([t-1] \Delta \theta) \Delta \theta \Delta \phi
\end{aligned}
$$

are often inaccurate, especially when the sampling is sparse (the antenna is electrically small). The lack accuracy is caused by the fact that the integral over $\theta$ does not involve a periodic spatially bandlimited function, so the trapezoidal rule is not guaranteed to work well [19, pages 111-113, 140-144, 372].

\section{Antenna Correlation in Isotropic Environment}

In this section we describe the concept of antenna correlation in an isotropic environment like the one observed in a reverberation chamber. However, first we state the classical definition of antenna correlation in terms of the plane-wave receiving characteristics introduced in Section 2.

Consider two receiving antennas, possibly mounted on the same DUT, with plane-wave receiving characteristics $\mathcal{R}_{1}(\theta, \phi)$ and $\mathcal{R}_{2}(\theta, \phi)$. The classical definition of the correlation $\rho$ between the two receiving antennas is

$$
\rho=\frac{\int_{0}^{2 \pi} \int_{0}^{\pi} \mathcal{R}_{1}(\theta, \phi) \cdot \mathcal{R}_{2}^{*}(\theta, \phi) \sin \theta d \theta d \phi}{\sqrt{\int_{0}^{2 \pi} \int_{0}^{\pi}\left|\mathcal{R}_{1}(\theta, \phi)\right|^{2} \sin \theta d \theta d \phi \int_{0}^{2 \pi} \int_{0}^{\pi}\left|\mathcal{R}_{2}(\theta, \phi)\right|^{2} \sin \theta d \theta d \phi}} .
$$

In accordance with Section 2, the correlation (13) can be computed by inserting Fourier series expansions for $\mathcal{R}_{1}(\theta, \phi)$ and $\mathcal{R}_{2}(\theta, \phi)$ with Fourier coefficients obtained from sampled values.

A general specification of the isotropic environment can be found in Hill's book [1, Section 7.1] and in the paper by De Doncker and Meys [11]. Here we consider a specific embodiment [5-9] involving plane waves propagating in a set of fixed directions. The points $\left(\theta_{s}, \phi_{s}\right), s=1,2, \ldots, S$, are roughly evenly distributed on the unit sphere as shown in Figure 3. More specifically, the points are on constant $-\theta$ rings with the number of points on each ring dependent on $\theta$. In particular, the top and bottom rings $\theta=0$ and $\theta=\pi$ consist of just one point each.

Assume that two plane waves are incoming in each of the directions $\left(\theta_{s}, \phi_{s}\right)$. One of them is $\theta$-polarized with amplitude 
$\alpha_{s}^{(j)}$; the other is $\phi$-polarized with amplitude $\beta_{s}^{(j)}$, where $j=$ $1,2, \ldots, J$. For a particular $j$, the incident field is thus given by

$$
\mathbf{E}^{(j)}(\mathbf{r})=\sum_{s=1}^{S}\left[\alpha_{s}^{(j)} \hat{\boldsymbol{\theta}}_{s} e^{-i k \hat{\mathbf{r}}_{s} \cdot \mathbf{r}}+\beta_{s}^{(j)} \hat{\boldsymbol{\phi}}_{s} e^{-i k \hat{\mathbf{r}}_{\mathbf{s}} \cdot \mathbf{r}}\right]
$$

where $\hat{\mathbf{r}}_{s}, \hat{\boldsymbol{\theta}}_{s}$, and $\hat{\boldsymbol{\phi}}_{s}$ are the spherical unit vectors evaluated at $(\theta, \phi)=\left(\theta_{s}, \phi_{s}\right)$. The plane-wave amplitudes $\alpha_{s}^{(j)}$ and $\beta_{s}^{(j)}$ are uniformly distributed independent complex random variables with zero mean, and the corresponding outputs of the two receiving antennas are

$$
\begin{aligned}
& V_{1}^{(j)}=\sum_{s=1}^{S}\left[\alpha_{s}^{(j)} \hat{\boldsymbol{\theta}}_{s} \cdot \boldsymbol{R}_{1}\left(\theta_{s}, \phi_{s}\right)+\beta_{s}^{(j)} \hat{\boldsymbol{\phi}}_{s} \cdot \boldsymbol{R}_{1}\left(\theta_{s}, \phi_{s}\right)\right], \\
& V_{2}^{(j)}=\sum_{s=1}^{S}\left[\alpha_{s}^{(j)} \hat{\boldsymbol{\theta}}_{s} \cdot \boldsymbol{R}_{2}\left(\theta_{s}, \phi_{s}\right)+\beta_{s}^{(j)} \hat{\boldsymbol{\phi}}_{s} \cdot \boldsymbol{R}_{2}\left(\theta_{s}, \phi_{s}\right)\right] .
\end{aligned}
$$

The isotropic environment is obtained as the collection of incident fields $\mathbf{E}^{(j)}(\mathbf{r})$ for $j=1,2, \ldots, J$, with a new set of amplitudes $\alpha_{s}^{(j)}$ and $\beta_{s}^{(j)}$ selected for each $j$. Thus, we can compute the outputs $V_{1}^{(j)}$ and $V_{2}^{(j)}$ for each of the two receiving antennas for $j=1,2, \ldots, J$. Section 5 presents MIMO capacity simulations with a receiving DUT placed in this isotropic environment.

It was shown by De Doncker and Meys [11] that the correlation between the outputs $V_{1}$ and $V_{2}$ in the isotropic environment is equal to the classical correlation (13):

$$
\rho=\operatorname{corr}\left(V_{1}, V_{2}\right) .
$$

We shall now demonstrate through numerical simulations that this result is indeed correct and investigate the sampling rate (density of incident plane waves) required in the isotropic environment to make (16) accurate.

3.1. Two Hertzian Dipoles. Consider two $z$-directed Hertzian dipoles on the $x$-axis at $\mathbf{r}_{1}=(-d / 2,0,0)$ and $\mathbf{r}_{2}=(d / 2,0,0)$ so that the radius of the minimum sphere is $R_{\min }=d / 2$; see Figure 4. The output of a Hertzian dipole is proportional to the incident electric field in the direction of the dipole. Hence,

$$
\begin{aligned}
& \mathcal{R}_{1}(\theta, \phi)=L \hat{\boldsymbol{\theta}} \sin \theta e^{i k(d / 2) \cos \phi \sin \theta}, \\
& \mathcal{R}_{2}(\theta, \phi)=L \hat{\boldsymbol{\theta}} \sin \theta e^{-i k(d / 2) \cos \phi \sin \theta},
\end{aligned}
$$

where $L$ is a constant length. The correlation between the dipoles is found from (13) to be

$$
\rho(d)=\frac{3}{2}\left[\frac{\sin (k d)}{k d}-\frac{1}{(k d)^{2}}\left(\frac{\sin (k d)}{k d}-\cos (k d)\right)\right] .
$$

Hill [1, equation (7.63)] confirms the general result (16) that the classical correlation (18) is the correlation obtained in an isotropic environment.

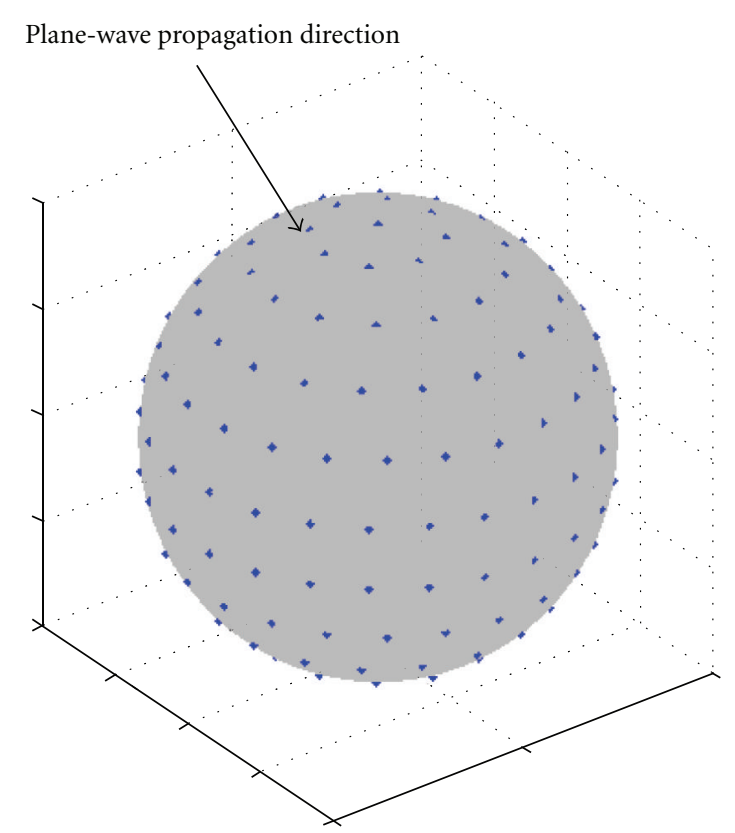

Figure 3: A selection of points $\left(\theta_{s}, \phi_{s}\right), s=1,2, \ldots, S$, evenly distributed on the unit sphere. Each point defines a direction of propagation for two plane waves. One plane wave is $\theta$-polarized, the other is $\phi$-polarized.

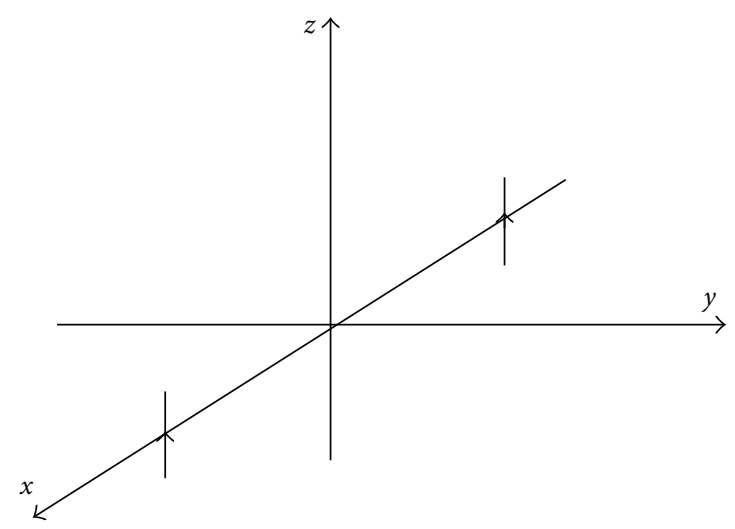

Figure 4: Two $z$-directed Hertzian dipoles on the $x$-axis at $\mathbf{r}_{1}=$ $(-d / 2,0,0)$ and $\mathbf{r}_{2}=(d / 2,0,0)$.

We compute the correlation from (16) for $d=\lambda / 2$ and $d=2 \lambda$ with varying $S$. Throughout, $J=10000$. As a measure of the density of incident plane waves, we use the "isotropic" spacing $\Delta_{i}$ between constant $\theta$ rings on the unit sphere. Specifically, if there are $\mathcal{N}$ constant $-\theta$ rings (including the two at $\theta=0$ and $\theta=\pi)$, we have $\Delta_{i}=\pi /(\mathcal{N}-1)$.

Unlike the points $\left(\theta_{t}, \phi_{p}\right)$ with $\Delta \theta$ and $\Delta \phi$ spacing used in computing the Fourier coefficients in Section 2, the points $\left(\theta_{s}, \phi_{s}\right)$ do not lie on a rectangular grid. Hence, the number of plane-wave directions of incidence in the isotropic environment (denoted by $S$ ) is smaller than the number of grid points used to compute the Fourier coefficients in Section 2, even if $\Delta_{i}=\Delta \theta$. 
TABle 1: Correlations computed from the classical formula (13) and from the isotropic simulation formula (16) with 180 plane-wave directions of propagation. The data is collected at $751 \mathrm{MHz}$.

\begin{tabular}{lccc}
\hline & Good antenna & Nominal antenna & Bad antenna \\
\hline Classical formula (13) & $-0.0381+0.0009 i$ & $-0.5749-0.0054 i$ & $-0.9042+0.0172 i$ \\
Isotropic simulation formula (16) & $-0.0367-0.0020 i$ & $-0.5740-0.0040 i$ & $-0.9066+0.0130 i$ \\
\hline
\end{tabular}

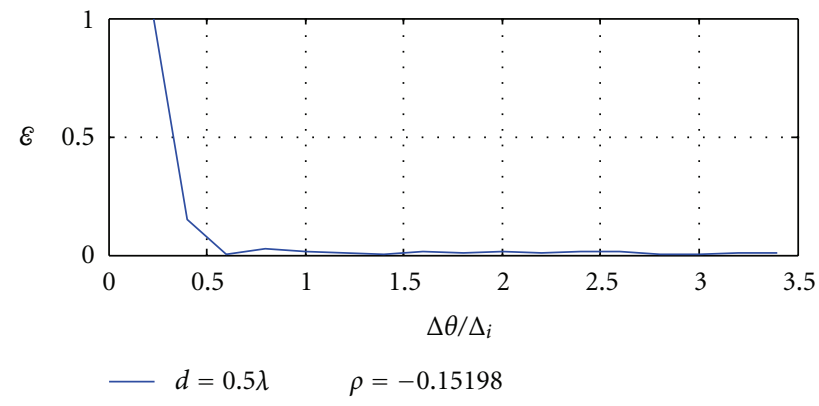

(a)

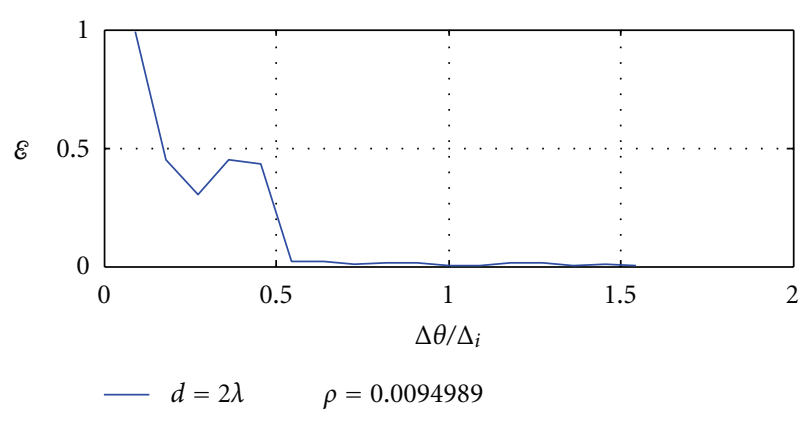

(b)

Figure 5: The error $\mathcal{E}$ in (19) as a function of $\Delta \theta / \Delta_{i}$ where $\Delta \theta=\pi /(N+1)$. $N$ is computed from (5) with $\gamma=2$. Large values of $\Delta \theta / \Delta_{i}$ correspond to dense plane-wave distributions in the isotropic environment.

Figure 5 shows the error:

$$
\mathcal{E}=\left|\rho(d)-\operatorname{corr}\left(V_{1}, V_{2}\right)\right|,
$$

where $\rho(d)$ is the exact correlation (18) and $\operatorname{corr}\left(V_{1}, V_{2}\right)$ is the correlation obtained from the isotropic environment, as a function of $\Delta \theta / \Delta_{i}$. We set $\gamma=2$ in the computation of $N$ in (5) and use $\Delta \theta=\pi /(N+1)$.

For both $d=\lambda / 2$ and $d=2 \lambda$, the error becomes negligible when the ratio $\Delta \theta / \Delta_{i}$ is about 0.7. In other words, accurate correlations are obtained when the isotropic sampling distance $\Delta_{i}$ is about 1.4 times the sampling distance $\Delta \theta$ required to compute the Fourier coefficients in Section 2 with $(\gamma=2)$. It is not surprising that accurate correlations are obtained in the isotropic environment when $\Delta_{i}>\Delta \theta$ since the correlation (13) is an "average-over-an-entiresphere" quantity whereas the expression for $\Delta \theta$ is derived to achieve the more demanding "point-by-point" accuracy.

To accurately reproduce the isotropic field conditions in a reverberation chamber, one must choose a chamber size large enough to ensure enough plane-wave directions of incidence for a given DUT size. We also note that these numerical simulations validate the general theorem by De Doncker and Meys [11].

Before leaving this section we investigate the sampling required for a $2 \mathrm{D}$ configuration where the correlation is based on incident fields from a small region of the unit sphere. This type of model will be used in Section 5 to simulate a transmitting base station that broadcasts according to a Laplacian distribution.

The two $z$-directed Hertzian dipoles are still on the $x$-axis at $\mathbf{r}_{1}=(-d / 2,0,0)$ and $\mathbf{r}_{2}=(d / 2,0,0)$ as shown in Figure 6 . The dipoles are now illuminated by a collection of plane waves that all propagate in the $x-y$ plane. 180 directions of incidence are selected according to the approximate Laplacian distribution [21, equation (18)] with $\sigma_{\theta}=35^{\circ}$ centered on $\phi=90^{\circ}$, as indicated in Figure 6. At any instant, each of the 180 plane waves is multiplied by a random phase to create a particular incident field. We achieve 10000 different incident fields by applying 10000 independent sets of random phases. The outputs $V_{1}^{(j)}$ and $V_{2}^{(j)}$ are thus obtained for $j=1,2, \ldots, 10000$, and the exact correlation in this experiment is $\operatorname{corr}\left(V_{1}, V_{2}\right)$.

We also compute an approximate correlation based on a fixed set of equally spaced directions of incidence illustrated by the ring in Figure 6 . The angular spacing between two directions of incidence on this ring is $\Delta_{r}$. We simply replace the directions of incidence from the Laplacian distribution by the closest direction of incidence on this ring. The sampling theorem derived in Section 2 states that the angular spacing between these directions of incidence should be $\Delta \phi=\pi /(N+$ 1) with $N$ computed from (5).

Figure 7 shows the error of the approximate correlation as a function of the ratio $\Delta \phi / \Delta_{r}$. We see that the error in this case with a limited range of directions of incidence only vanishes when $\Delta_{r}$ is roughly equal to the spacing $\Delta \phi$ required by the sampling theorem. Hence, to accurately reproduce the model-specified field conditions in an anechoic-chamber tests system consisting of a ring of antennas, one must supply enough antennas to satisfy the sampling theorem.

3.2. CTIA Reference Antennas. To expedite the baseline between laboratories participants of CTIA LTE round robin, a set of MIMO $2 \times 2$ reference antennas has been developed [10]. A subset of these antennas has dimension $240 \times 80 \times$ $1 \mathrm{~mm}$ and operate at $751 \mathrm{MHz}$ corresponding to $R_{\min }=$ $0.12 \mathrm{~m}$ and $k R_{\min }=1.89$. Hence, (5) gives $N=4$ when $\gamma=$ 2 , and the required sampling is therefore $\Delta \theta=\Delta \phi=36^{\circ}$.

We compute the isotropic correlation with 180 planewave directions of propagation corresponding to an isotropic sampling of $\Delta_{i}=16.3^{\circ}$. Table 1 shows the resulting 


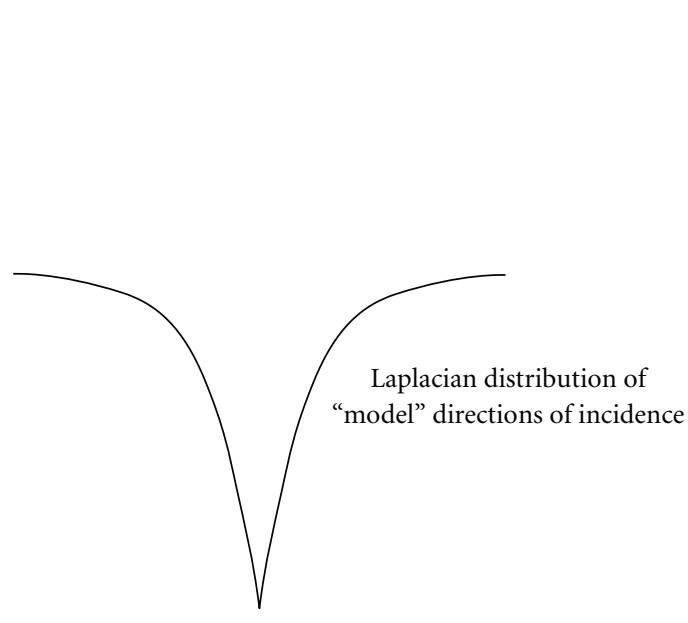

(a)

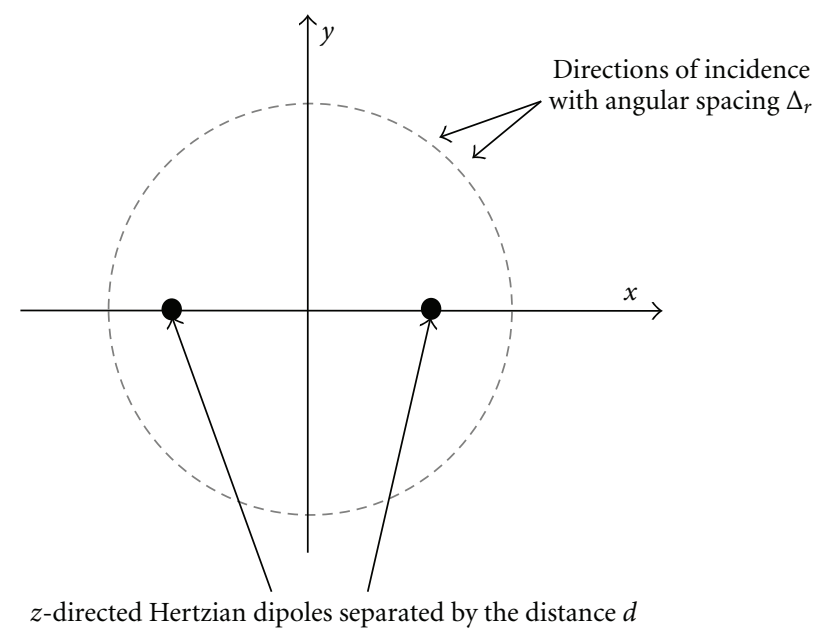

(b)

Figure 6: Two $z$-directed Hertzian dipoles on the $x$-axis at $\mathbf{r}_{1}=(-d / 2,0,0)$ and $\mathbf{r}_{2}=(d / 2,0,0)$ with surrounding plane-wave directions of propagation and Laplacian distribution. All directions of incidence are in the $x-y$ plane.

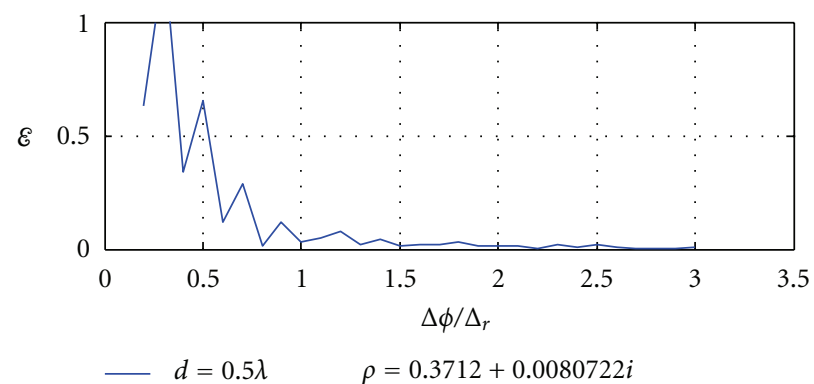

(a)

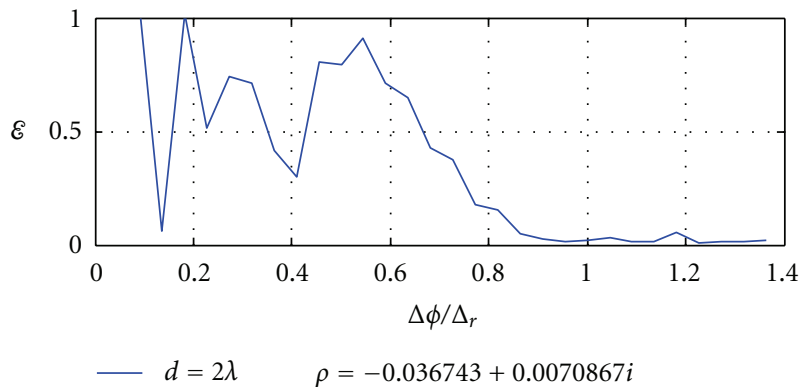

(b)

Figure 7: The error $\mathcal{E}$ as a function of $\Delta \phi / \Delta_{r}$ where $\Delta \phi=\pi /(N+1) . N$ is computed from (5) with $\gamma=2$. Large values of $\Delta \phi / \Delta_{r}$ correspond to dense plane-wave distributions along in the ring in Figure 6.

correlations for three antennas: (i) good antenna with low correlation, (ii) nominal antenna with average correlation, and (iii) bad antenna with high correlation. The table validates the general theorem by De Doncker and Meys [11] in (16).

These results have also been verified experientially in a reverberation chamber at NIST [5-9].

\section{Isotropic Environment of a Rectangular Reverberation Chamber}

A reverberation chamber provides a rich scattering environment that is ideal for over-the-air testing of wireless devices. The chamber typically contains a number of wall-mounted transmitting antennas, a mechanical mode stirrer, and a turntable on which the DUT is placed; see Figure 8. The turntable provides so-called platform stirring [22]. As we shall see, at any given position of stirrer and turntable, the DUT is illuminated by a large number of plane waves whose directions of propagation are determined by the modes of the chamber $[1,2]$. We assume that the chamber is excited by a Hertzian dipole with frequency $f$ (as usual $\omega=2 \pi f$ ).

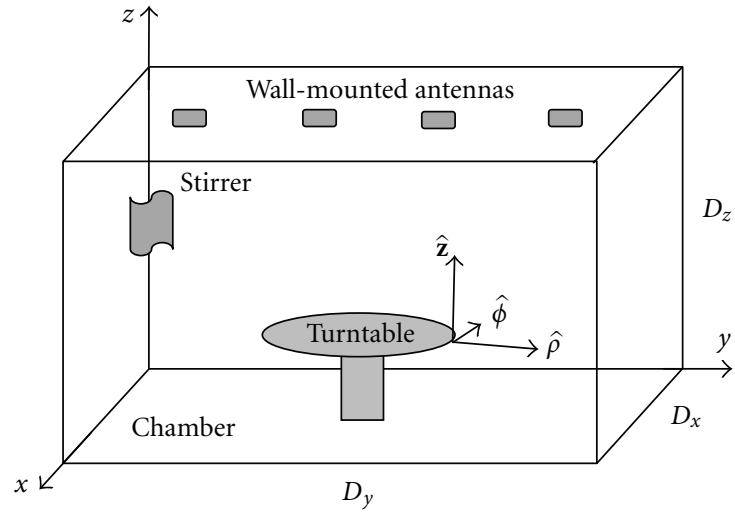

Figure 8: Rectangular reverberation chamber with dimensions $D_{x}$, $D_{y}$, and $D_{z}$. The chamber contains four wall-mounted transmitting antennas, a mechanical stirrer, and a turntable. The DUT position is near the edge of the turntable.

4.1. Modes in Terms of Plane Waves. Let the rectangular chamber have the dimensions $D_{x}, D_{y}$, and $D_{z}$ as shown in Figure 8 . The dyadic Green's function (field due to a Hertzian 
dipole source) can be expressed as a superposition of modes determined such that the tangential electric field vanishes on the chamber walls [3, pages 383-384]. Specifically, each rectangular field component can be expressed as a sum of terms of the form (we use the $z$ component for illustration purposes; the $x$ and $y$ components have similar expressions):

$$
\begin{aligned}
& E_{z}\left(x, y, z, x^{\prime}, y^{\prime}, z^{\prime}, n_{x}, n_{y}, n_{z}\right) \\
& =E_{0}\left(x^{\prime}, y^{\prime}, z^{\prime}, n_{x}, n_{y}, n_{z}\right) \\
& \quad \times \sin \left(\frac{n_{x} \pi x}{D_{x}}\right) \sin \left(\frac{n_{y} \pi y}{D_{y}}\right) \cos \left(\frac{n_{z} \pi z}{D_{z}}\right),
\end{aligned}
$$

where $E_{0}\left(x^{\prime}, y^{\prime}, z^{\prime}, n_{x}, n_{y}, n_{z}\right)$ is independent of the observation point $(x, y, z)$ but dependent on the source location $\left(x^{\prime}, y^{\prime}, z^{\prime}\right)$, the source strength, and the mode indices $n_{x}, n_{y}$, and $n_{z}$, which can take on any nonnegative value.

There is one additional term (called an irrotational mode) that goes with the mode in (20) to ensure that the field satisfies the wave equation with wave number that corresponds to the medium in the chamber. The irrotational mode has an identical plane-wave representation, so analyzing (20) is sufficient. Also, the sum over $n_{z}$ can be performed in closed form to obtain a formula that involves just a double sum [3, page 384] and [1, page 34]. As usual, all losses (including wall losses) are accounted for by an effective lossy medium [3, page 389] and [1, page 35].

We associate a frequency $f_{n_{x}, n_{y}, n_{z}}$ and a propagation constant $k_{n_{x}, n_{y}, n_{z}}$ with each mode:

$$
\begin{aligned}
f_{n_{x}, n_{y}, n_{z}} & =\frac{c}{2} \sqrt{\left(\frac{n_{x}}{D_{x}}\right)^{2}+\left(\frac{n_{y}}{D_{y}}\right)^{2}+\left(\frac{n_{z}}{D_{z}}\right)^{2}}, \\
k_{n_{x}, n_{y}, n_{z}} & =\sqrt{\left(\frac{n_{x} \pi}{D_{x}}\right)^{2}+\left(\frac{n_{y} \pi}{D_{y}}\right)^{2}+\left(\frac{n_{z} \pi}{D_{z}}\right)^{2}} \\
& =\sqrt{k_{x}^{2}+k_{y}^{2}+k_{z}^{2}},
\end{aligned}
$$

where $k_{x}=n_{x} \pi / D_{x}, k_{y}=n_{y} \pi / D_{y}$, and $k_{z}=n_{z} \pi / D_{z}$. Note that $\sin (x)=\left(e^{i x}-e^{-i x}\right) /(2 i)$ and $\cos (x)=\left(e^{i x}+e^{-i x}\right) / 2$ to convert (20) to

$$
\begin{aligned}
E_{z}\left(x, y, z, x^{\prime}, y^{\prime}, z^{\prime}, n_{x}, n_{y}, n_{z}\right) & \\
=- & \frac{E_{0}\left(x^{\prime}, y^{\prime}, z^{\prime}, n_{x}, n_{y}, n_{z}\right)}{8} \\
& \times\left[e^{i\left(k_{x} x+k_{y} y+k_{z} z\right)}+7 \text { more terms }\right],
\end{aligned}
$$

where "7 more terms" indicate that the square bracket contains seven additional terms of the form $e^{i\left( \pm k_{x} x \pm k_{y} y \pm k_{z} z\right)}$. Hence, each mode can be expressed as the sum of eight plane waves with propagation vectors $\pm k_{x} \widehat{\mathbf{x}} \pm k_{y} \hat{\mathbf{y}} \pm k_{z} \hat{\mathbf{z}}$. The excitation factor can be written as

$$
E_{0}\left(x^{\prime}, y^{\prime}, z^{\prime}, n_{x}, n_{y}, n_{z}\right)=\frac{F_{0}\left(x^{\prime}, y^{\prime}, z^{\prime}, n_{x}, n_{y}, n_{z}\right)}{(1-2 i / Q) f_{n_{x}, n_{y}, n_{z}}^{2}-f^{2}},
$$

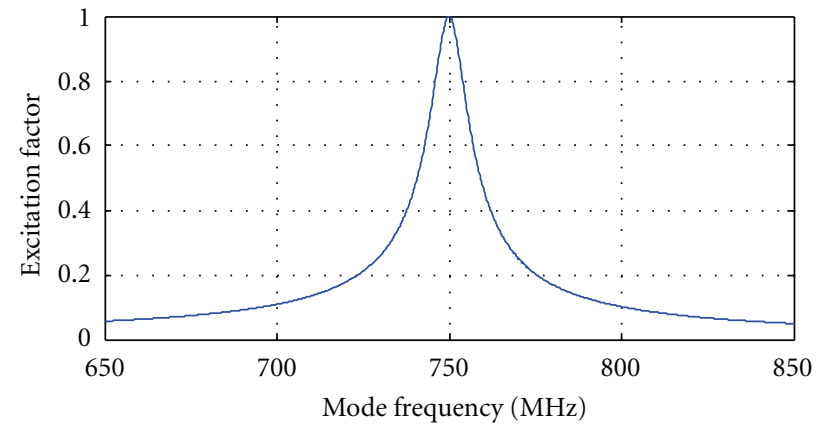

Figure 9: Normalized magnitude of the excitation factor as function of mode frequency for a $750 \mathrm{MHz}$ driving signal and a 30 ns RMS delay. Only modes in a " $3 \mathrm{~dB}$ " region near the peak get effectively excited.

where $F_{0}\left(x^{\prime}, y^{\prime}, z^{\prime}, n_{x}, n_{y}, n_{z}\right)$ is independent of frequency $f$ and $Q$ is the quality factor that accounts for wall and other losses in the chamber. $Q$ is related to the RMS power delay time $\tau$ through $\tau=Q / \omega$. The 8 directions of incidence for a single mode are

$$
\widehat{\mathbf{k}}^{i}=\frac{ \pm \hat{\mathbf{x}}\left(n_{x} / D_{x}\right) \pm \hat{\mathbf{y}}\left(n_{y} / D_{y}\right) \pm \hat{\mathbf{z}}\left(n_{z} / D_{z}\right)}{\sqrt{\left(n_{x} / D_{x}\right)^{2}+\left(n_{y} / D_{y}\right)^{2}+\left(n_{z} / D_{z}\right)^{2}}}
$$

with the corresponding mode frequency given by (21).

The magnitude of the excitation factor $1 /[(1-$ 2i/Q) $f_{n_{x}, n_{y}, n_{z}}^{2}-f^{2}$ ] (normalized) as a function of mode frequency $f_{n_{x}, n_{y}, n_{z}}$ for a $750 \mathrm{MHz}$ driving signal and a $30 \mathrm{~ns}$ RMS delay is shown in Figure 9. We see that this factor has a peak at the diving frequency and that it falls off fairly slowly away from this frequency. For a chamber to work well, it must support a significant number of plane waves, which translates into the requirement that there must be a significant number of modes in the region where the excitation factor is significantly nonzero. One typically sets the threshold point where modes are considered "unexcitable" at the point where the excitation factor has fallen $3 \mathrm{~dB}$. Notice that the width of the excitation factor depends on the quality factor. One of the benefits of using multiple wall-mounted transmitting antennas is that all excitable modes do actually get excited.

Let us now show the actual plane-wave directions of incidence for two reverberation chambers with $30 \mathrm{~ns}$ RMS delay that are driven by a $750 \mathrm{MHz}$ source. One is electrically large $\left(D_{x}=3 \mathrm{~m}, D_{y}=2 \mathrm{~m}\right.$, and $\left.D_{z}=1.5 \mathrm{~m}\right)$ at $750 \mathrm{MHz}$; the other is electrically small $\left(D_{x}=1 \mathrm{~m}, D_{y}=0.9 \mathrm{~m}\right.$, and $\left.D_{z}=0.8 \mathrm{~m}\right)$ at $750 \mathrm{MHz}$. We include modes that lie in a $50 \mathrm{MHz}$ band around $750 \mathrm{MHz}$. Figure 10 (a) shows the plane-wave directions of incidence for the large chamber. The directions of incidence are nonuniformly distributed over the unit sphere with a maximum distance between points of $22^{\circ}$ and an average distance of $6^{\circ}$. The largest gaps occur near the north and south poles.

As the stirrer in the reverberation chamber rotates, the amplitudes and phases of the plane waves change to produce an isotropic environment as discussed in the previous 


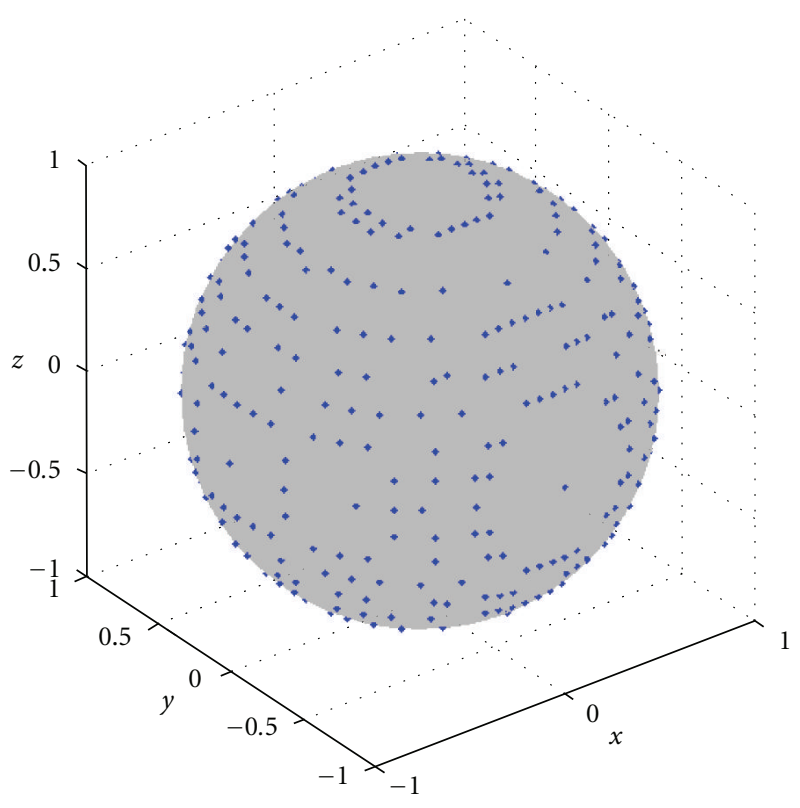

(a)

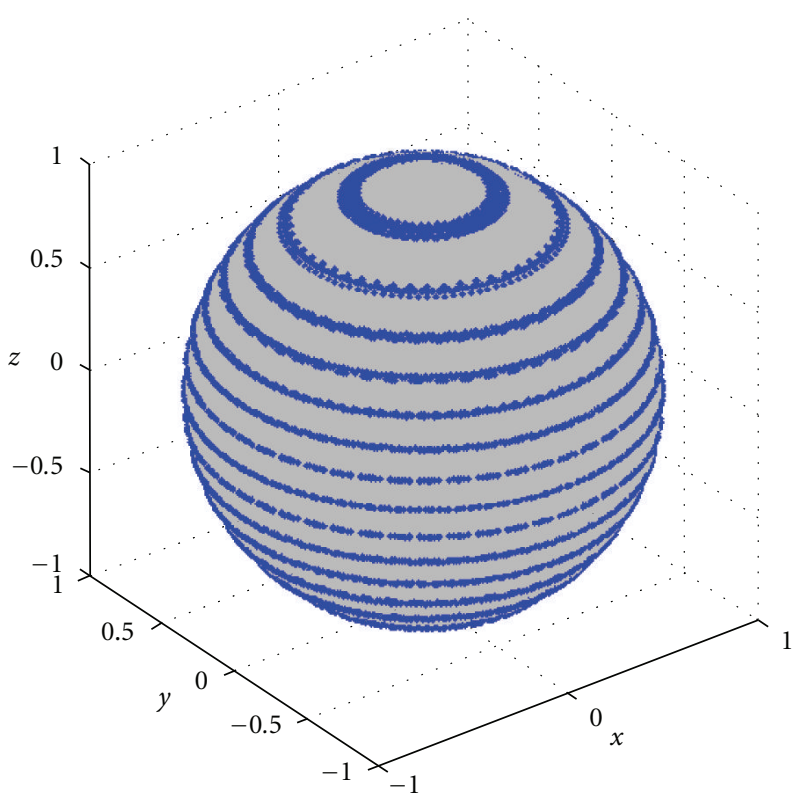

(b)

Figure 10: Plane-wave directions of incidence for modes in a $50 \mathrm{MHz}$ band around $750 \mathrm{MHz}$ in a large chamber with dimensions $D_{x}=3 \mathrm{~m}$, $D_{y}=2 \mathrm{~m}$, and $D_{z}=1.5 \mathrm{~m}$. (a) Without turntable. (b) With turntable rotating around a $z$-directed axis. Without the turntable, the maximum distance between directions of incidence is $22^{\circ}$ and the average distance is $6^{\circ}$.

section. In addition to the stirrer, the chamber contains a turntable on which the DUT rides. As the turntable rotates, the DUT sees the plane waves from different angles, effectively creating additional directions of incidence. Figure 10(b) shows all the directions of incidence (see from the point of view of the DUT) for a turntable that rotates around a $z$-directed axis, as illustrated in Figure 8. The turntable thus multiplies the effective number of plane waves available. Of course, only plane waves corresponding to a single rotated version of Figure 10(a) are available at any instant.

Figure 11(a) shows the plane-wave directions of incidence for the small chamber. The directions of incidence are sparsely distributed with a maximum distance between points of $48^{\circ}$ and an average distance of $27^{\circ}$. There are large gaps throughout the unit sphere. Figure 11(b) shows all the directions of incidence (see from the point of view of the DUT) for the turntable in Figure 8. Again, only plane waves corresponding to a single rotated version of Figure 11(a) are available at any instant.

4.2. Correlation Simulations. Let us now investigate how these reverberation chambers perform when evaluating the correlation between the two $z$-directed Hertzian dipoles in Figure 4 . The exact correlation is given by (18), and the error $\mathcal{E}$ in (19) for the large chamber is shown in Figure 12 as a function of dipole separation. The "turntable off" simulation is performed by randomly changing the phase and amplitude of incident plane waves with directions of incidence in Figure $10(a)$ (the plane waves are given by $\mathbf{E}_{0} \exp \left(-i k \widehat{\mathbf{k}}^{i} \cdot \mathbf{r}\right.$ ) with $\hat{\mathbf{k}}^{i}$ given by (25) and $\hat{\mathbf{k}}^{i} \cdot \mathbf{E}_{0}=0$ ). The "turntable on" simulation is performed by repeating the "turntable off" simulation with the plane-wave directions of incidence rotated by a fixed amount. In other words, the "turntable on" simulation accurately replicates the actual situation encountered by the DUT in a real reverberation chamber where only one set of plane waves (corresponding to a rotated version of the plane waves for a fixed DUT orientation) is available at any given instant. Figure 12 demonstrates that the turntable dramatically improves the accuracy of the computed correlation between the dipoles. This improved accuracy is achieved by illuminating the two dipoles from additional directions.

The error $\mathcal{E}$ in (19) for the small chamber is shown in Figure 13 as a function of dipole separation. With the sparse coverage of plane-wave directions of incidence (Figure 11(a)), the correlation obtained with the turntable off is very inaccurate. The error is above 0.3 for certain values of the dipole separation. Remarkably, with the turntable on, the error falls dramatically to an almost tolerable level.

4.3. Anisotropy Coefficients. One of the most important statistical properties of the field in the chamber is the degree to which it is isotropic, that is, how evenly distributed are the directions of propagation and polarizations of the incoming plane waves at the location of the DUT. With the isotropy test developed by the international standards committee (ICE) [23], field anisotropy coefficients measure the bias of the average direction of polarization of the electric field in the chamber. The bias is computed by comparing three components of the electric field obtained from dipole-antenna measurements. The degree of isotropy 


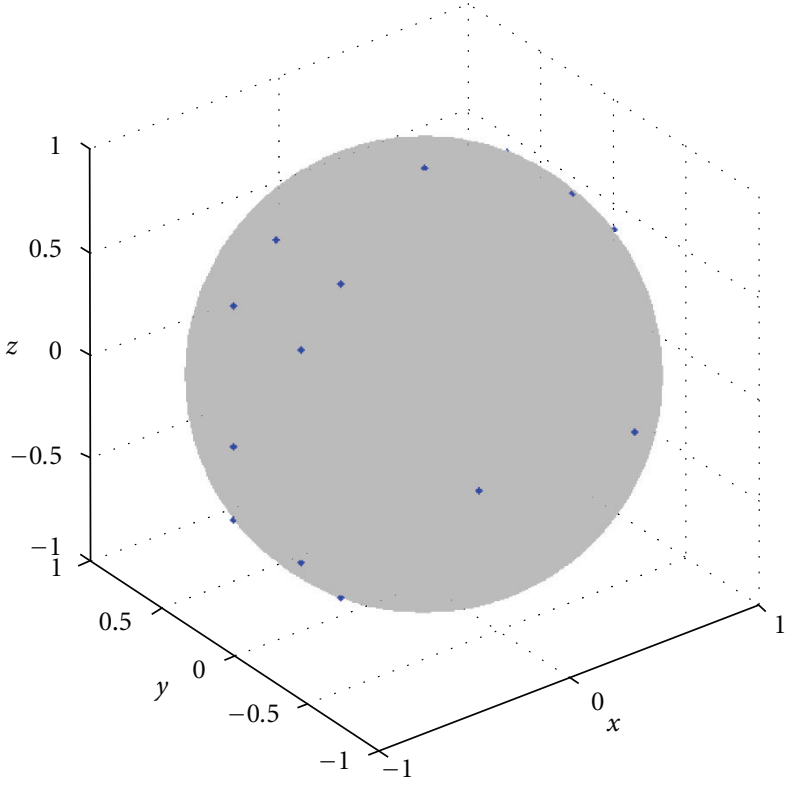

(a)

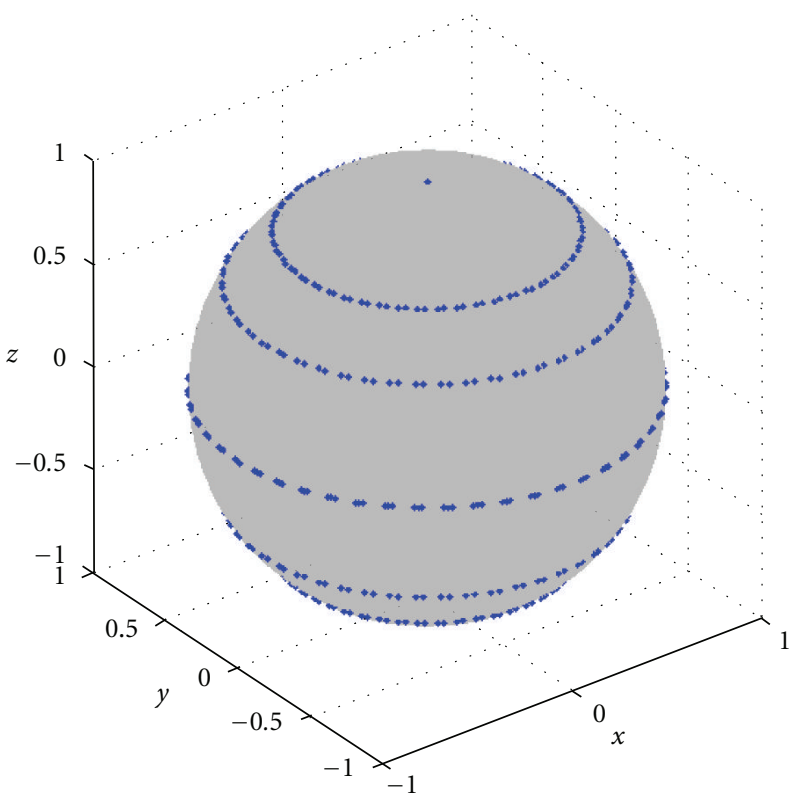

(b)

FIgURE 11: Plane-wave directions of incidence for modes in a $50 \mathrm{MHz}$ band around $750 \mathrm{MHz}$ in a small chamber with dimensions $D_{x}=1$ m, $D_{y}=0.9 \mathrm{~m}$, and $D_{z}=0.8 \mathrm{~m}$. (a) Without turntable. (b) With turntable rotating around a $z$-directed axis. Without the turntable, the maximum distance between directions of incidence is $48^{\circ}$ and the average distance is $27^{\circ}$.

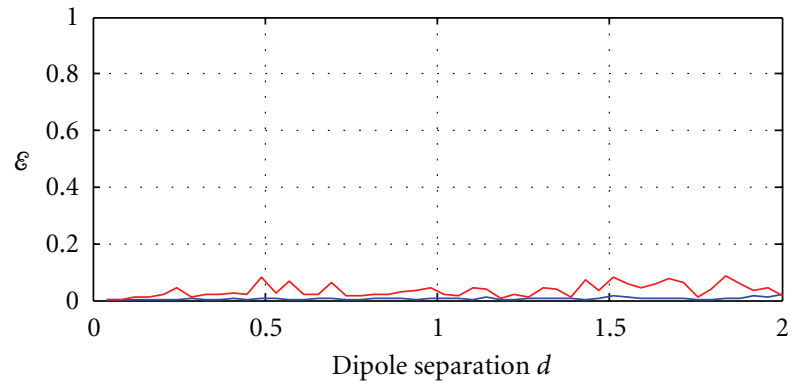

— Turntable on

_ Turntable off

FIgURE 12: The error $\mathcal{E}$ as a function of dipole separation $d$ for the large chamber with dimensions $D_{x}=3 \mathrm{~m}, D_{y}=2 \mathrm{~m}$, and $D_{z}=$ $1.5 \mathrm{~m}$ operating around $750 \mathrm{MHz}$.

of an actual chamber is determined by comparing the observed and ideal (known from theory) distributions of the anisotropy coefficients. We shall next compute the anisotropy coefficients for the large and small reverberation chambers introduced above.

Three orthogonal components of the electric field recorded at the location of the DUT are required to compute the field anisotropy coefficients [23]:

$$
A_{\alpha \beta}(n)=\frac{\left|E_{\alpha}(n)\right|^{2} / P_{i}(n)-\left|E_{\beta}(n)\right|^{2} / P_{i}(n)}{\left|E_{\alpha}(n)\right|^{2} / P_{i}(n)+\left|E_{\beta}(n)\right|^{2} / P_{i}(n)},
$$

where $\alpha$ and $\beta$ denote the three orthogonal directions. The quantity $P_{i}(n)$ is the net input power (forward minus

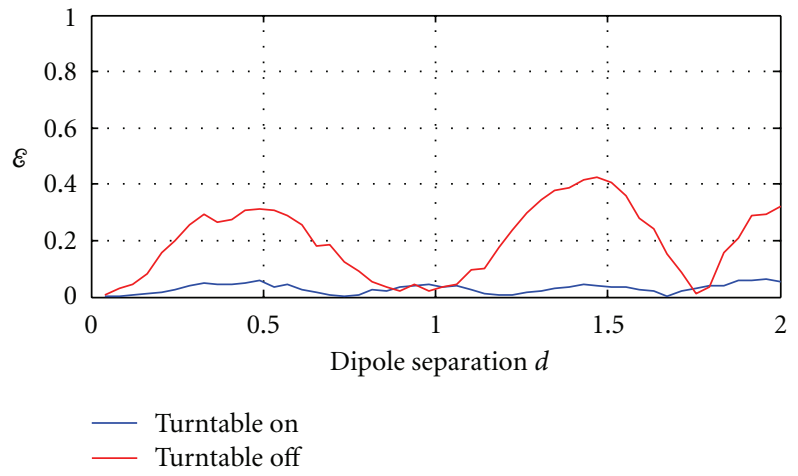

FIGURE 13: The error $\varepsilon$ as a function of dipole separation $d$ for the small chamber with dimensions $D_{x}=1 \mathrm{~m}, D_{y}=0.9 \mathrm{~m}$, and $D_{z}=$ $0.8 \mathrm{~m}$ operating around $750 \mathrm{MHz}$.

reflected) injected into the chamber, and $n$ is an index that determines the position of the turntable, the position of the mechanical stirrer, and the active transmit antenna. In addition to the three anisotropy coefficients defined by (26), the test in [23] also employs a total anisotropy coefficient that we shall not investigate here. For a perfectly isotropic chamber, $A_{\alpha \beta}(n)$ is uniformly distributed between -1 and 1. Pages 195 and 196 of [23] show plots of anisotropy coefficients for well-stirred and poorly stirred reverberation chambers.

When the DUT is placed near the edge of the turntable in Figure 8, the three relevant orthogonal directions for the isotropy test are (i) the direction $\hat{\mathbf{z}}$ normal to the turn table, (ii) the direction $\hat{\phi}$ tangential to the edge of the turntable, and (iii) the direction $\hat{\boldsymbol{\rho}}$ radial to the turntable. Hence, in a 

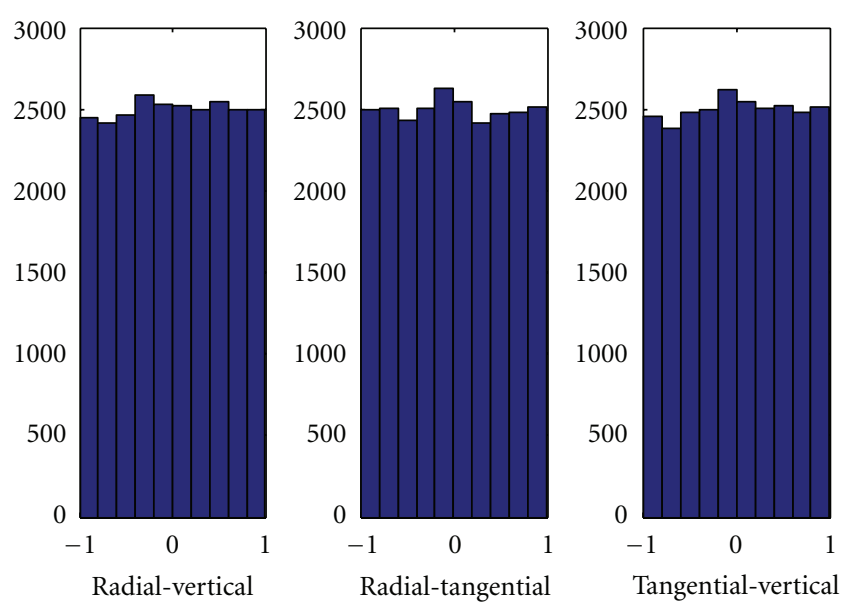

FIGURE 14: Histograms of anisotropy coefficients for large chamber with dimensions $D_{x}=3 \mathrm{~m}, D_{y}=2 \mathrm{~m}$, and $D_{z}=1.5 \mathrm{~m}$ operating around $750 \mathrm{MHz}$.
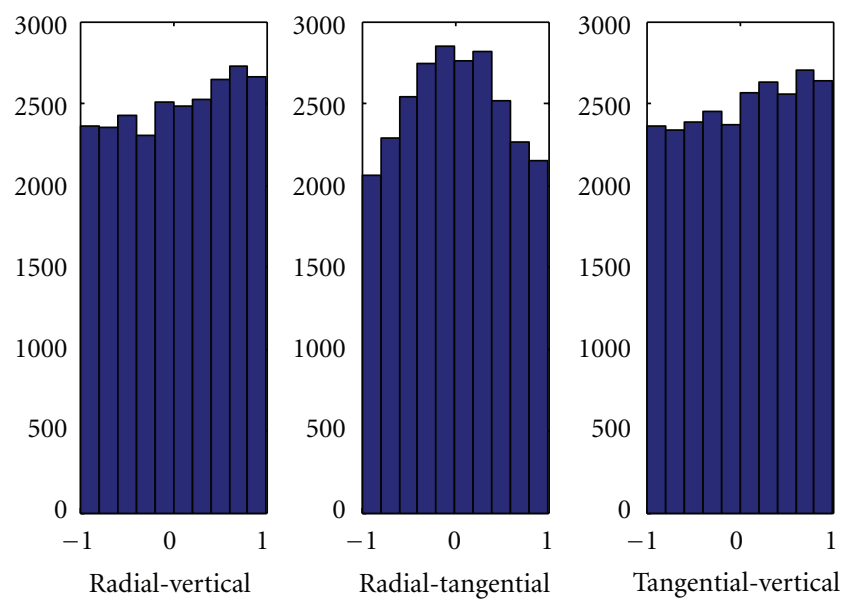

FIGURE 15: Histograms of anisotropy coefficients for small chamber with dimensions $D_{x}=1 \mathrm{~m}, D_{y}=0.9 \mathrm{~m}$, and $D_{z}=0.8 \mathrm{~m}$ operating around $750 \mathrm{MHz}$.

standard cylindrical coordinate system $(\rho, \phi, z)$ centered on the turntable, $\alpha$ and $\beta$ can take the values $\rho$, $\phi$, and $z$.

Figures 14 and 15 show the histograms for the anisotropy coefficients of the large $\left(D_{x}=3 \mathrm{~m}, D_{y}=2 \mathrm{~m}\right.$, and $D_{z}=$ $1.5 \mathrm{~m})$ and small $\left(D_{x}=1 \mathrm{~m}, D_{y}=0.9 \mathrm{~m}\right.$, and $\left.D_{z}=0.8 \mathrm{~m}\right)$ reverberation chambers (both with the turntable active). We see that the anisotropy coefficients for the large chamber closely follow a uniform distribution, whereas the anisotropy coefficients for the small chamber show a significant deviation from a uniform distribution. We conclude that the small chamber does not produce enough plane waves, even though the turntable is active.

4.4. Remarks. We have now described the plane-wave environment in a reverberation chamber using the mode expansion of the dyadic Green's function for the rectangular box. We have seen how the plane-wave directions of incidence as seen from the DUT depend on the dimensions of the chamber and on whether or not a turntable is active. Through numerical simulations, we evaluated the accuracy of correlation experiments in the chamber. It would be nice to have a theory that explicitly determined the accuracy of the chamber as a function of chamber dimension and chamber loading (quality factor $Q$ ). Such a theory does not exist, and at the present moment the accuracy can only be determined by numerical simulations like the ones presented here. Moreover, if a precise theory existed, it would have to be fairly complicated because accuracy depends not only on mode density (which is largely determined by chamber volume [1, page 30]) but also on the actual plane-wave directions of incidence, which depend on all 3 rectangular chamber dimensions. For example, a large chamber may have a large "plane-wave gap" near the poles, which in turn can make it less accurate than a smaller chamber.

\section{MIMO Capacity Simulations}

In this section we perform MIMO capacity simulations with a two-antenna DUT receiver in an isotropic environment. The transmitter is a standard two-antenna base station. We employ the good, nominal, and bad reference antennas described in Section 3.2 and show that they produce clearly distinguishable throughput curves. The approach taken in this section was inspired by [5-9].

A schematic of the channel model is shown in Figure 16. The base station employs two antennas separated by $D=4 \lambda$ and radiating through a standard 3-sector pattern $\mathcal{A}(\Theta)[24$, page 9]. The voltage input amplitudes for the two base station antennas are denoted $V_{1}^{T}$ and $V_{2}^{T}$. Each base station antenna broadcasts in the directions $\Theta_{s}, s=1,2, \ldots, S$, according to the Laplacian distribution approximation [21, equation (18)] with $\sigma_{\theta}=5^{\circ}$. We have now $S$ complex numbers for base station antenna 1 ,

$$
U_{s}^{(1)}=V_{1}^{T} \mathcal{A}\left(\Theta_{s}\right) e^{i k(D / 2) \sin \Theta_{s}},
$$

and $S$ complex numbers for base station antenna 2,

$$
U_{s}^{(2)}=V_{2}^{T} \mathcal{A}\left(\Theta_{s}\right) e^{-i k(D / 2) \sin \Theta_{s}} .
$$

In practice, the two-antenna 2D Laplacian base station output would be fed to the reverberation chamber through two or more wall antennas. The directions of propagation from the base station are thus distributed randomly into plane waves in the chamber, and the Laplacian distribution is not preserved. In other words, the chamber does not reproduce the Laplacian distribution. However, the correlation imposed on the two information streams by the base station is preserved. Further, one often feeds a reverberation chamber from a channel emulator that is programmed to produce advanced spatial channel models, which can include both Doppler spectra, long time delays (much longer than the one produced by the chamber alone), and specified directions of incidence. When such channel models are fed to the reverberation chamber, the channel model is said to be evaluated isotropically. In such situations, the specified directions of propagation dictated by the channel model are not preserved. However, if the emulator and reverberation 
chamber are adjusted properly, the time delays and Doppler spectra of the channel model are preserved in the chamber. The use of advanced channel models adds a lot of flexibility to the reverberation chamber as an over-the-air test tool.

Next we select a set of evenly distributed points $\left(\theta_{s}, \phi_{s}\right)$, $s=1,2, \ldots, S$, on the unit sphere as described in Section 3 to obtain $S$ incoming plane-wave propagation directions for illuminating the DUT. For $j=1,2, \ldots, J$ we compute a set of random permutations of the numbers $1,2, \ldots, S$ given by $s^{\prime}=p(j, s)$. In addition, for $j=1,2, \ldots, J$, we compute two sets of uniformly distributed random variables $v_{\theta}(j, s)$ and $v_{\phi}(j, s)$ in the range from 0 to $2 \pi$. The permutations $s^{\prime}=p(j, s)$ facilitate the random pairing between point on the base station pattern and plane-wave directions of incidence. The variables $v_{\theta}(j, s)$ and $v_{\phi}(j, s)$ provide random phase adjustments for each pair.

We now have $J$ different propagation channels that result in the following DUT antenna outputs:

$$
\begin{aligned}
V_{1}^{(j)}=\sum_{s=1}^{S}[ & \left.U_{p(j, s)}^{(1)}+U_{p(j, s)}^{(2)}\right] \\
& \times\left[e^{i v_{\theta}(j, s)} \hat{\boldsymbol{\theta}}_{s} \cdot \boldsymbol{R}_{1}\left(\theta_{s}, \phi_{s}\right)+e^{i v_{\phi}(j, s)} \hat{\boldsymbol{\phi}}_{s} \cdot \mathcal{R}_{1}\left(\theta_{s}, \phi_{s}\right)\right], \\
V_{2}^{(j)}=\sum_{s=1}^{S}[ & \left.U_{p(j, s)}^{(1)}+U_{p(j, s)}^{(2)}\right] \\
& \times\left[e^{i v_{\theta}(j, s)} \hat{\boldsymbol{\theta}}_{s} \cdot \boldsymbol{R}_{2}\left(\theta_{s}, \phi_{s}\right)+e^{i v_{\phi}(j, s)} \hat{\boldsymbol{\phi}}_{s} \cdot \mathcal{R}_{2}\left(\theta_{s}, \phi_{s}\right)\right],
\end{aligned}
$$

which can be written in matrix form as

$$
\left[\begin{array}{c}
V_{1}^{(j)} \\
V_{2}^{(j)}
\end{array}\right]=\overline{\mathbf{H}}^{(j)}\left[\begin{array}{c}
V_{1}^{T} \\
V_{2}^{T}
\end{array}\right],
$$

where $\overline{\mathbf{H}}^{(j)}$ is the $2 \times 2$ channel matrix. The corresponding channel capacities are

$$
C^{(j)}=\log _{2}\left[\operatorname{det}\left(\overline{\mathbf{I}}+\frac{P_{t}}{2 N_{0}} \overline{\mathbf{H}}^{(j)}\left(\overline{\mathbf{H}}^{(j)}\right)^{H}\right)\right],
$$

where the superscript $H$ indicates the transpose complex conjugate, $N_{0}$ is the receiver noise, and $P_{t}$ the transmitter power.

Figure 17 shows the capacity curves as functions of the signal to noise ratio (SNR) for the good, nominal, and bad reference antennas computed with $S=180$ and $J=10000$. These curves are obtained by computing the mean capacity for varying SNR. The mean is taken over all $J=10000$ propagation channels. To achieve a capacity of $7 \mathrm{bps} / \mathrm{Hz}$, the three reference antennas require very different SNR values: the bad reference antenna requires an SNR of $19 \mathrm{~dB}$, whereas the good reference antenna requires only an SNR of $13 \mathrm{~dB}$. The difference in SNR between good and bad reference antennas is in this case $6 \mathrm{~dB}$. Similarly, to achieve a capacity of $12.5 \mathrm{bps} / \mathrm{Hz}$, the difference in SNR between good and bad reference antennas is $7 \mathrm{~dB}$. Hence, the capacity curves are clearly distinguishable, thereby confirming the capability of the isotropic environment for over-the-air MIMO testing. However, given the large variation in correlation of the three

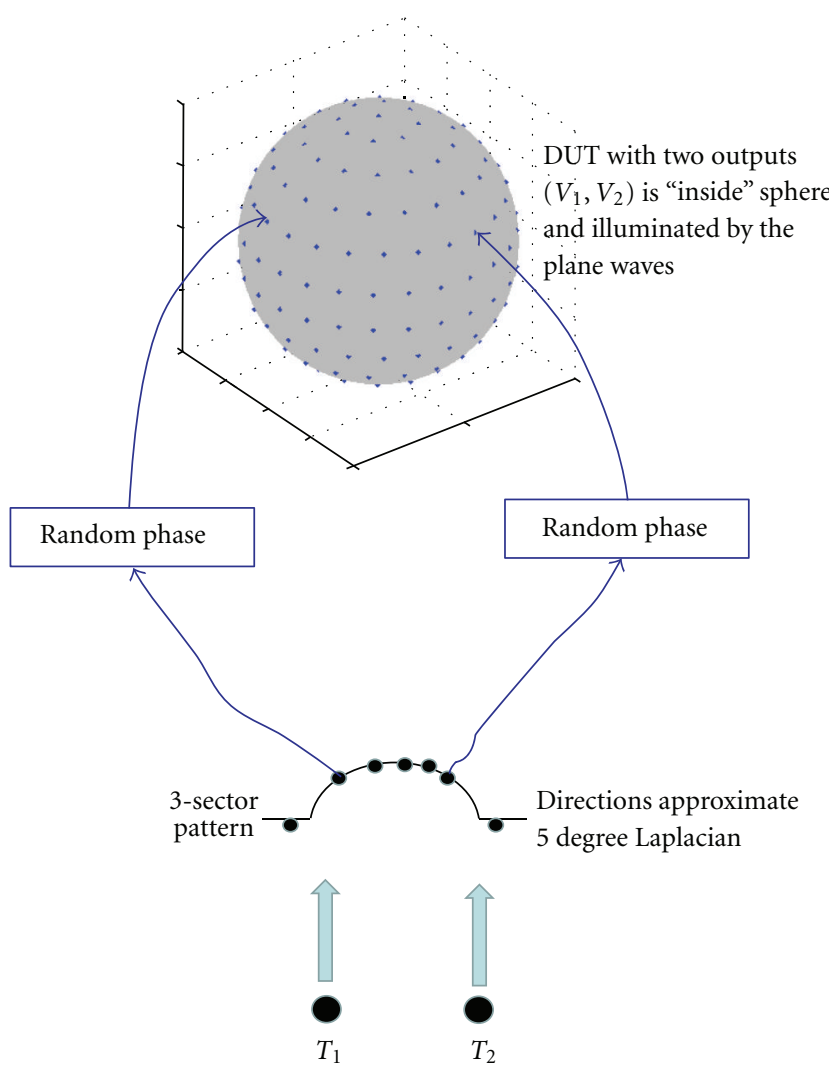

Base station with $4 \lambda$ separation

FIGURE 16: Channel model employing an isotropic environment. Each point on the base station pattern is randomly paired with a plane-wave propagation direction illuminating the DUT. In addition, each "pairing" is supplied by a random phase. The multiple states of the model are obtained by changing the pairing and the random phases.

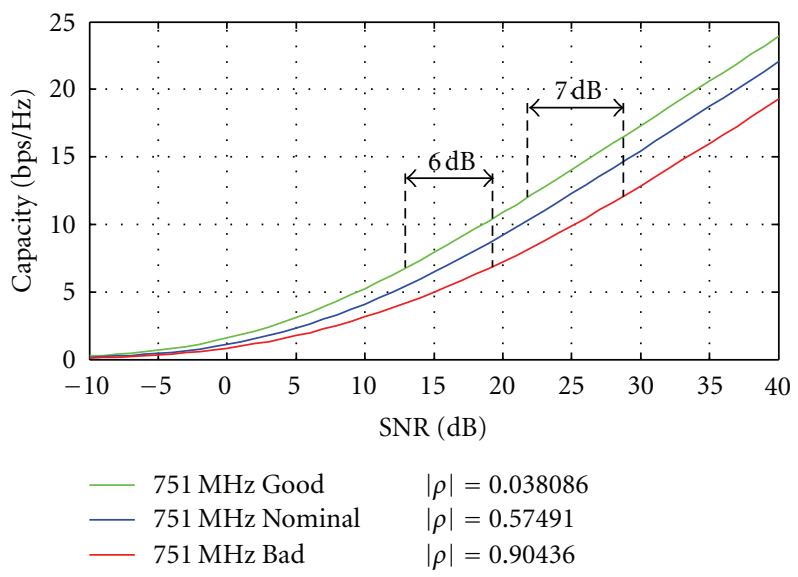

Figure 17: Capacity curves as functions of SNR for the "good," "nominal," and "bad" reference antennas in an isotropic environment. To achieve a capacity of $7 \mathrm{bps} / \mathrm{Hz}$, the three reference antennas require very different SNR values: the bad reference antenna requires an SNR of $19 \mathrm{~dB}$, whereas the good reference antenna requires only an SNR of $13 \mathrm{~dB}$. The difference in SNR between good and bad reference antennas is in this case $6 \mathrm{~dB}$. Similarly, to achieve a capacity of $12.5 \mathrm{bps} / \mathrm{Hz}$, the difference in SNR between good and bad reference antennas is $7 \mathrm{~dB}$. 
antennas (see Table 1), it is no surprise that the capacity curves are very different. Most practical antennas would likely fall in the nominal category.

\section{Conclusions}

We investigated the use of reverberation chambers for overthe-air testing of MIMO devices by examining antenna correlation and throughput in isotropic environments. A truncated spherical-wave expansion was used to derive sampling theorems and the Fourier expansions for the pattern of an arbitrary DUT. The required sampling rate of the pattern depends on the frequency, the physical size of the entire DUT (not just its antenna), and the relative location of the DUT to the spherical coordinate system.

Through numerical investigations involving Hertzian dipoles, it was shown how the sampling rate for the pattern determines the plane-wave density required in the isotropic environment to obtain accurate values for the correlation between antennas. It was also demonstrated that antenna correlation in the isotropic environment is equivalent to the classical definition of antenna correlation, as was proven theoretically by De Doncker and Meys [11]. In particular, the correlations computed for the CTIA reference antennas [10] in isotropic simulations agree with the correlations obtained from the classical definition.

Using the dyadic Green's function for the rectangular box, we computed the plane-wave distribution for realistic reverberation chambers, which were in turn used in simulations of antenna correlations and anisotropy coefficients. No general theory that explicitly determined the accuracy of the chamber as a function of chamber dimension and chamber loading was found. Instead we explained how one can determine accuracy estimates through simulations.

We performed MIMO channel capacity simulations using a standard base station model and the DUT (employing the CTIA reference antennas) placed in a single-tap plane-wave reverberation chamber model. The capacity curves obtained with the good, nominal, and bad reference antennas were clearly distinguishable, as would be expected given the vast difference between the correlations of these antennas; see Table 1.

Hence, we conclude that isotropic tests performed in a reverberation chamber can distinguish between DUTs that employ the different CTIA reference antennas. It would be interesting to perform link-level simulations with multitap isotropic channel models to further investigate this over-theair test method.

\section{Acknowledgments}

The author would like to thank R. J. Pirkl and K. A. Remley of the NIST for numerous helpful discussions. I. Szini of Motorola is thanked for providing the patterns for the reference antennas.

\section{References}

[1] D. A. Hill, Electromagnetic Fields in Cavities, John Wiley \& Sons, New York, NY, USA, 2009.
[2] K. Rosengren and P. S. Kildal, "Study of distributions of modes and plane waves in reverberation chambers for the characterization of antennas in a multipath environment," Microwave and Optical Technology Letters, vol. 30, no. 6, pp. 386-391, 2001.

[3] R. E. Colin, Field Theory of Guided Waves, IEEE Press, New York, NY, USA, 2nd edition, 1991.

[4] Azimuth Systems, "Isotropy and rayleighness of emulatorchamber system," White Paper, 2011.

[5] R. J. Pirkl and K. A. Remley, "A study of channel capacity in 2-D and 3-D isotropic environments," CTIA Contribution MOSG110805, 2011.

[6] R. J. Pirkl and K. A. Remley, "A study of channel capacity in 2-D and 3-D isotropic environments: comparison between MIMO reference antennas," CTIA Contribution MOSG110906, 2011.

[7] R. J. Pirkl and K. A. Remley, "A study of channel capacity in 2D and 3-D isotropic environments: effect of gain imbalance," CTIA Contribution MOSG111008, 2011.

[8] R. J. Pirkl, E. Engvall, and K. A. Remley, "Measurements of correlation coefficients and capacity CDFs for MIMO reference antennas," CTIA Contribution MOSG111009, 2011.

[9] R. J. Pirkl and K. A. Remley, "Dependence of antenna orientation on correlation, average gain, and capacity in 2-D isotropic environments," CTIA Contribution MOSG111010, 2011.

[10] I. Szini, "Reference antenna proposal for MIMO OTA," CTIA Contribution MOSG110504R1, 2011.

[11] P. De Doncker and R. Meys, "Statistical response of antennas under uncorrelated plane wave spectrum illumination," Electromagnetics, vol. 24, no. 6, pp. 409-423, 2004.

[12] T. B. Hansen and A. D. Yaghjian, Plane-Wave Theory of TimeDomain Fields, IEEE Press, New York, NY, USA, 1999.

[13] D. M. Kerns, "Plane-wave scattering-matrix theory of antennas and antenna-antenna interactions," NBS Monograph 162, U.S. Government Printing Office, Washington, DC, USA, 1981.

[14] W. W. Hansen, "A new type of expansion in radiation problems," Physical Review, vol. 47, no. 2, pp. 139-143, 1935.

[15] J. D. Jackson, Classical Electrodynamics, John Wiley \& Sons, New York, NY, USA, 2nd edition, 1975.

[16] W. C. Chew, J. M. Jin, E. Michielssen, and J. Song, Eds., Fast and Efficient Algorithms in Computational Electromagnetics, Artech-House, Boston, Mass, USA, 2006.

[17] L. J. Ricardi and M. L. Burrows, "A recurrence technique for expanding a function in spherical harmonics," IEEE Transactions on Computers, vol. 21, no. 6, pp. 583-585, 1972.

[18] P. F. Wacker, "Non-planar near-field measurements: spherical scanning," NBS Internal Report 75-809, 1975.

[19] J. E. Hansen, J. Hald, F. Jensen, and F. H. Larsen, Eds., Spherical Near-Field Antenna Measurements, Peter Peregrinus, London, UK, 1988.

[20] T. B. Hansen, "Formulation of spherical near-field scanning for electromagnetic fields in the time domain," IEEE Transactions on Antennas and Propagation, vol. 45, no. 4, pp. 620-630, 1997.

[21] M. Patzold and Q. Yao, "A study of stochastic and deterministic procedures for the sedign of simulation models for spatial channels," in Proceedings of the IEEE International Symposium on Personal, Indoor and Mobile Radio Communications (PIMRC '02), pp. 1924-1931, September 2002.

[22] K. Rosengren, P. S. Kildal, C. Carlsson, and J. Carlsson, "Characterization of antennas for mobile and wireless terminals in reverberation chambers: improved accuracy by platform 
stirring," Microwave and Optical Technology Letters, vol. 30, no. 6, pp. 391-397, 2001.

[23] International Standard, Testing and measurement techniquesReverberation chamber test methods, IEC 61000-4-21, 2003.

[24] 3rd Generation Partnership Project, Technical Specification Group Radio Access Network, "Spatial channel model for Multiple Input Multiple Output (MIMO) simulations," 3GPP TR 25.996 V8.0.0, 2008. 

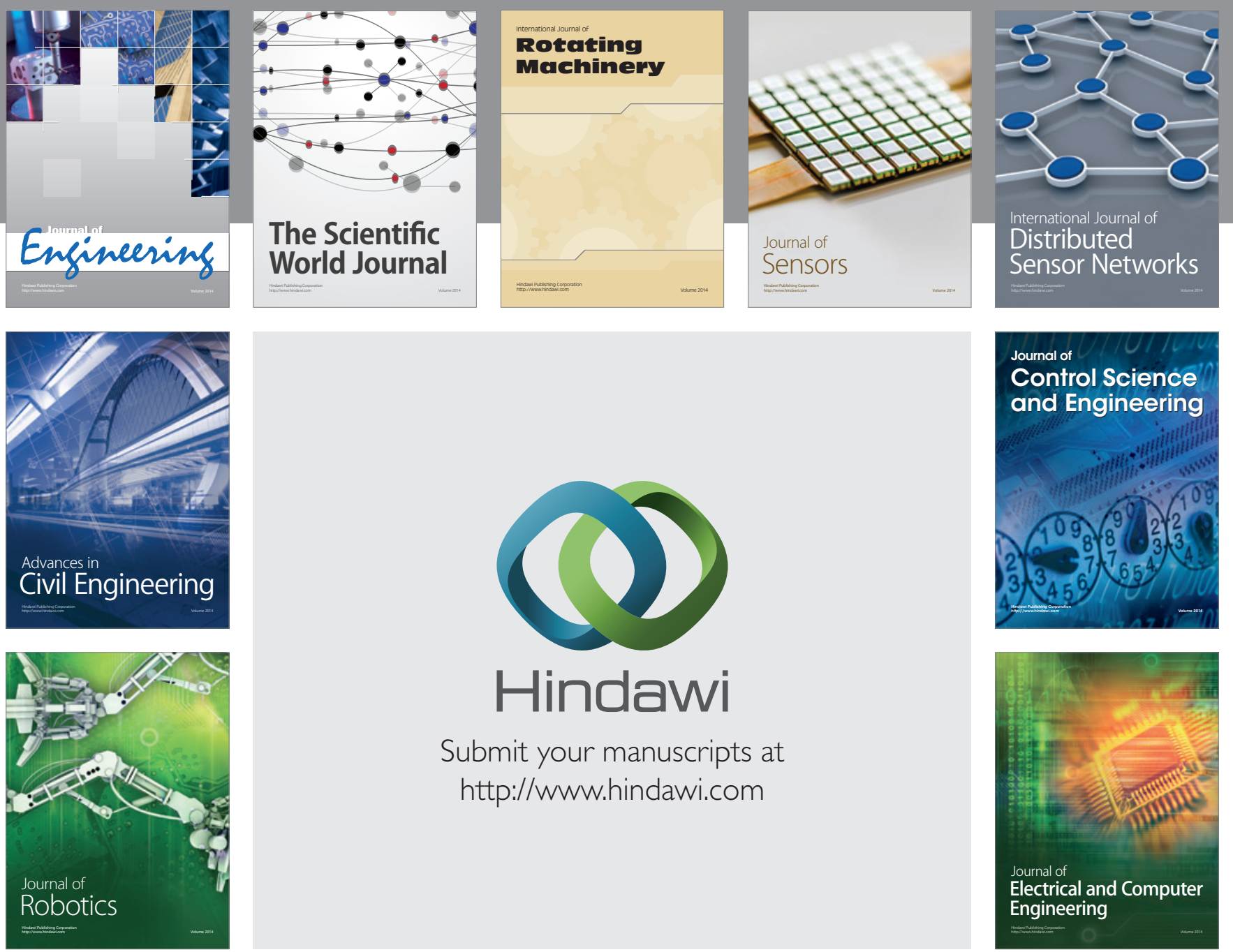

Submit your manuscripts at

http://www.hindawi.com
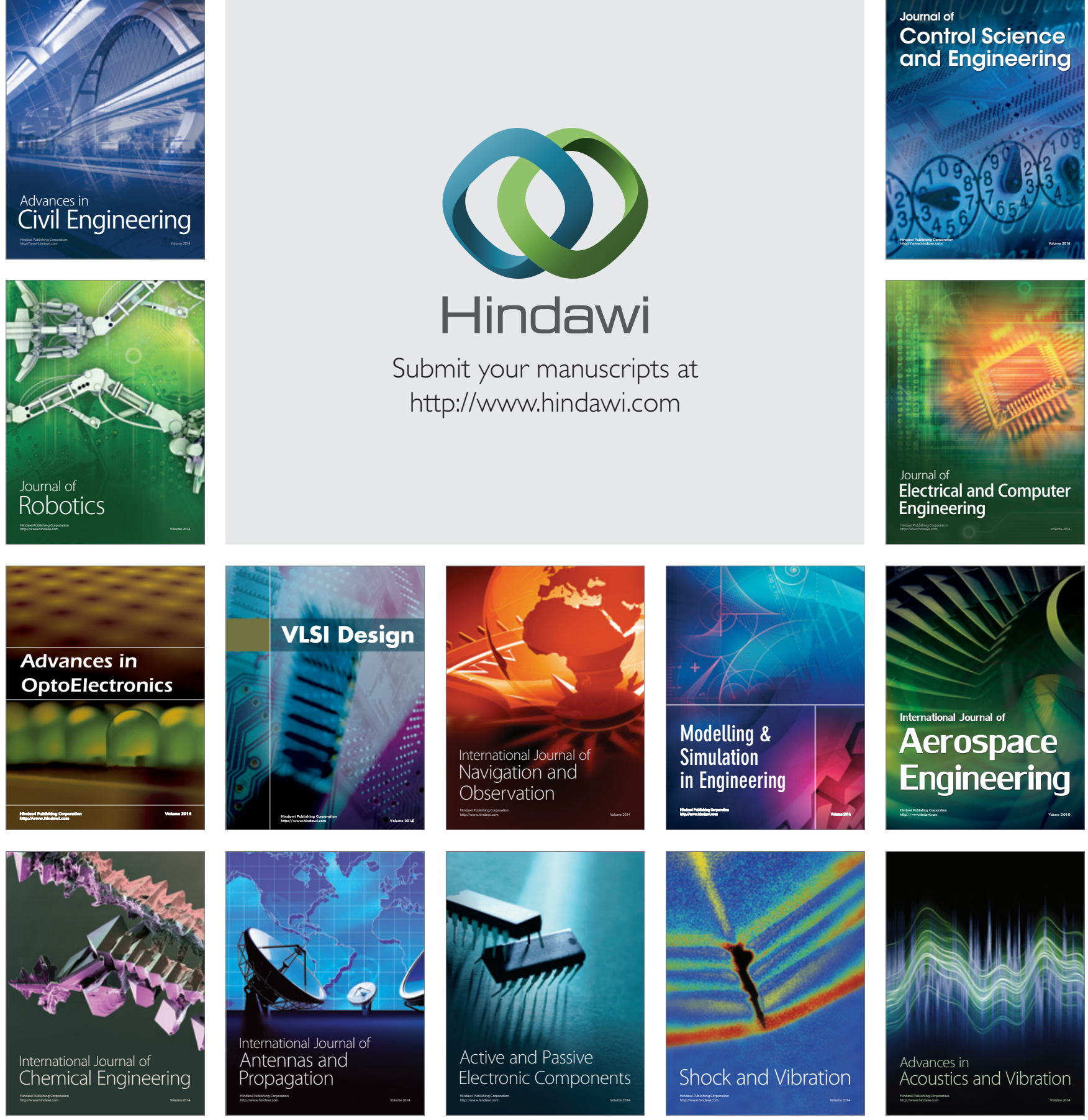